\title{
The formation and evolution of bright spots on Ceres
}

\author{
N.T. Stein ${ }^{\mathrm{a}, *}$, B.L. Ehlmann ${ }^{\mathrm{a}, \mathrm{b}}$, E. Palomba ${ }^{\mathrm{c}, \mathrm{d}}$, M.C. De Sanctis ${ }^{\mathrm{c}}, \mathrm{A}$. Nathues ${ }^{\mathrm{e}}$, H. Hiesinger ${ }^{\mathrm{f}}$, \\ E. Ammannito ${ }^{g}$, C.A. Raymond ${ }^{\text {b }}$, R. Jaumann ${ }^{\mathrm{h}}$, A. Longobardo ${ }^{\mathrm{d}}$, C.T. Russell ${ }^{\mathrm{g}}$ \\ a Division of Geological and Planetary Sciences, California Institute of Technology, Pasadena, CA 91125, United States \\ bet Propulsion Laboratory, California Institute of Technology, Pasadena, CA 91109, United States \\ ' INAF-IAPS, Via del Fosso del Cavaliere 100, 00133 Rome, Italy \\ ${ }^{\mathrm{d}}$ ASDC-ASI, Via del Politecnico, 00133 Rome, Italy \\ ${ }^{\mathrm{e}}$ Max Planck Institute for Solar System Research, Justus-von-Liebig-Weg 3, 37077 Goettingen, Germany \\ ${ }_{\mathrm{f}}^{\mathrm{f}}$ Institut für Planetologie, Westfälische Wilhelms-Universität. Münster, Germany \\ ${ }^{g}$ Department of Earth, Planetary, and Space Sciences, University of California, Los Angeles, CA 90095, United States \\ ${ }^{\mathrm{h}}$ DLR, Institute of Planetary Research, Rutherfordstraße 2, 12489 Berlin, Germany
}

\section{A R T I C L E I N F O}

\section{Article history:}

Received 12 May 2017

Revised 27 September 2017

Accepted 12 October 2017

Available online 6 November 2017

\section{Keywords:}

Asteroid Ceres

Impact processes

Surface

Faculae

\begin{abstract}
A B S T R A C T
The otherwise homogeneous surface of Ceres is dotted with hundreds of anomalously bright, predominantly carbonate-bearing areas, termed "faculae," with Bond albedos ranging from $\sim 0.02$ to $>0.5$. Here, we classify and map faculae globally to characterize their geological setting, assess potential mechanisms for their formation and destruction, and gain insight into the processes affecting the Ceres surface and near-surface. Faculae were found to occur in four distinct geological settings, associated predominantly with impact craters: (1) crater pits, peaks, or floor fractures (floor faculae), (2) crater rims or walls (rim/wall faculae), (3) bright ejecta blankets, and (4) the mountain Ahuna Mons. Floor faculae were identified in eight large, deep, and geologically young (asteroid-derived model (ADM) ages of $<420 \pm 60 \mathrm{Ma}$ ) craters: Occator, Haulani, Dantu, Ikapati, Urvara, Gaue, Ernutet, and Azacca. The geometry and geomorphic features of the eight craters with floor faculae are consistent with facula formation via impact-induced heating and upwelling of volatile-rich materials, upwelling/excavation of heterogeneously distributed subsurface brines or their precipitation products, or a combination of both processes. Rim/wall faculae and bright ejecta occur in and around hundreds of relatively young craters of all sizes, and the geometry of exposures is consistent with facula formation via the excavation of subsurface bright material, possibly from floor faculae that were previously emplaced and buried. A negative correlation between rim/wall facula albedo and crater age indicates that faculae darken over time. Models using the Ceres crater production function suggest initial production or exposure of faculae by large impacts, subsequent dissemination of facula materials to form additional small faculae, and then burial by impact-induced lateral mixing, which destroys faculae over timescales of less than $1.25 \mathrm{Gyr}$. Cumulatively, these models and the observation of faculae limited to geologically young craters indicate relatively modern formation or exposure of faculae, indicating that Ceres' surface remains active and that the near surface may support brines in the present day.
\end{abstract}

(c) 2017 The Authors. Published by Elsevier Inc

This is an open access article under the CC BY license. (http://creativecommons.org/licenses/by/4.0/)

\section{Introduction}

Ceres' surface is rather dark. The average geometric albedo measured by Dawn's Visible and Infrared spectrometer (VIR) at $0.55 \mu \mathrm{m}$ is $0.094 \pm 0.008$, and the average bond albedo measured with the Framing Camera (FC) clear filter is $0.034 \pm 0.001$ (Ciarniello et al., 2017; Ammannito et al., 2016; Li et al., 2016).

\footnotetext{
* Corresponding author.

E-mail address: nstein@caltech.edu (N.T. Stein).
}

Ceres' dark surface materials, whose reflectance ranges between $\sim 0.03$ and 0.37 (Nathues et al., 2016), are a mixture of magnesium phyllosilicates, ammoniated phases, other low-albedo materials such as organic carbon and iron-bearing opaque phases, and small amounts of carbonates (King et al., 1992; De Sanctis et al., 2015; Ammannito et al., 2016). FC images show that the otherwise uniform-albedo dark surface is punctuated by bright regions associated primarily with impact craters. Here we use the term "facula" to refer to isolated bright spots, whereas more extensive bright regions surrounding impact craters are referred to as "bright ejecta." The material comprising faculae and bright ejecta is some- 
times referred to as "bright material." The brightest and most extensive of these regions, Cerealia Facula and Vinalia Faculae, are located in the central pit and floor of Occator crater and were visible upon Dawn's approach to Ceres (Nathues et al., 2015, 2017a). Cerealia Facula has an average Bond albedo of $0.24 \pm 0.01$ (Li et al., 2016), and its spectra are consistent with a composition that includes significantly more abundant carbonate of different composition than the average surface (Na-rich instead of $\mathrm{Ca}$, Mg-rich) as well as ammoniated salts such as $\mathrm{NH}_{4} \mathrm{Cl}$ or $\mathrm{NH}_{4} \mathrm{HCO}_{3}$ (De Sanctis et al., 2016). At least two faculae contiguous with VIR detections of $\mathrm{H}_{2} \mathrm{O}$ ice have been identified at latitudes higher than $30^{\circ} \mathrm{N}$ (Combe et al., 2016, 2017). A dedicated study of the composition of the faculae indicates that most of them are rich in $\mathrm{Mg}$ - or $\mathrm{Na}-$ carbonate (Carrozzo et al., 2017; Palomba et al., 2017), and thus they cannot be explained by meteoritic infall as is the case for some anomalously dark materials on Vesta (which would be considered brighter than average on the much darker surface of Ceres) (McCord et al., 2012; Reddy et al., 2012). Instead an endogenous origin is likely, perhaps due to the upwelling and crystallization of salts from brines or entrained altered solids that were brought to the surface (De Sanctis et al., 2016; Nathues et al., 2017b).

Since Dawn's initial investigation of Occator crater, the FC has imaged the entire illuminated surface at a resolution of $\sim 35 \mathrm{~m} /$ pixel in clear filter. These images have revealed the presence of hundreds of faculae and bright ejecta associated with impact craters. By characterizing the mechanisms by which bright materials are emplaced, we can better understand the composition and structure of the subsurface and the processes that shape Ceres' surface. Here we detail a comprehensive global mapping of bright materials using high resolution FC data and a new classification scheme to characterize their geologic setting. Results are used to investigate potential mechanisms for the formation and destruction of faculae and the processes that modify them over time.

\section{Identification and mapping of faculae}

Faculae were identified using a photometrically corrected clear filter HAMO FC-based albedo mosaic and defined as regions with a Bond albedo at least 30\% higher than that of surrounding pixels. The threshold of $30 \%$ was selected because it represents a typical spread of albedos about the mean for a given scene. Hence, an albedo 30\% higher than the local average represents twice the typical variation in a scene. When reported, the area of faculae was computed on the basis of the total number of pixels surpassing this threshold. This is similar to the criterion adopted by Palomba et al. (2017) to identify faculae in VIR images. Bond albedo measures the fractional power of electromagnetic radiation incident on a surface that is scattered back into space, whereas the geometric albedo is the ratio of surface brightness at zero phase angle to that of an idealized, flat Lambertian (diffusely scattering) surface. The Bond albedo is dominated by light scattered at a moderate range of phase angles well sampled by Dawn, and is hence less model-dependent than the geometric albedo, which relies on additional modeling assumptions due to the lack of some observations at small phase angles.

The Dawn FC has an angular resolution of $93.7 \mu \mathrm{rad} /$ pixel (Sierks et al., 2011), yielding image pixel scales of up to $\sim 35 \mathrm{~m}$ at Ceres. With the clear filter, Dawn FC is sensitive to wavelengths of light between $\sim 400$ and $1000 \mathrm{~nm}$ and peaking near $700 \mathrm{~nm}$ (Sierks et al., 2011). Because faculae on Ceres can be small and are often distributed at sub-km scales, FC images offer means to identify small faculae based on albedo. Faculae were not mapped north or south of $\sim 55^{\circ}$ due to the difficulty of distinguishing true faculae from bright reflections on sun-facing crater rims. In the future, candidate faculae in these regions may be identified on the basis of an improved photometric correction that incorporates high

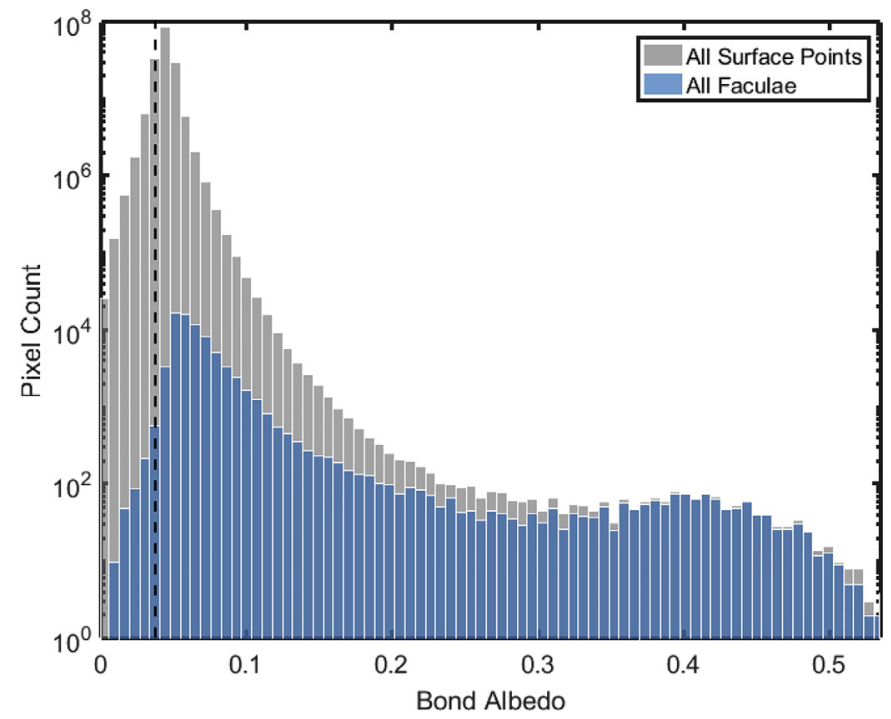

Fig. 1. Comparison of the Bond albedo of all faculae and all points on the surface as seen by FC. Faculae are identified based on the difference in albedo compared to the surrounding region, as discussed in the text. The vast majority of surface points with Bond albedos $>0.1$ that are not classified as faculae are due to reflections on sun-facing crater walls. The average Bond albedo of the surface is $0.034 \pm 0.001$ (dashed line) (Li et al., 2016). The peak near 0.4 is due to Cerealia Facula in Occator crater.

resolution topographic information. Faculae were confirmed visually in order to remove spurious identifications. The facula catalog was cross-referenced with a VIR-based facula catalog produced by Palomba et al. (2017), although not all faculae identified in FC images were verified with VIR spectra to contain Na carbonates because many FC-identified faculae are below the spatial resolution of VIR. That is, the defining characteristic of faculae reported here is their albedo rather than a specific composition. Although Palomba et al. (2017) also identify faculae on the basis of albedo, the higher spatial resolution of the FC LAMO data ( $35 \mathrm{~m} /$ pixel) relative to VIR LAMO data $(\sim 90-110 \mathrm{~m} /$ pixel) means the catalog presented here (included in the Supplementary materials) reports more faculae, particularly spatially small rim/wall faculae.

Faculae were mapped onto a LAMO FC clear filter mosaic of the surface by morphologic type. Facula extent was mapped using the 30\% threshold and tracked in a GIS database. Reported crater depths associated with some faculae were calculated from a photogrammetric FC digital elevation map (DEM), available in the PDS, as the average rim-to-floor depth of two transects in N-S and E-W directions.

\section{Distribution and classification of faculae}

There are at least 300 faculae or clusters of faculae in total, $>200$ of which are located on crater rims or walls. The albedo of the facula regions ranges from slightly below the average surface Bond albedo of $0.034 \pm 0.001$ to more than 0.5 in the central pit of Occator crater (Fig. 1) (Li et al., 2016). The lower portion of the range is set by the albedos of faculae adjacent to anomalously dark material or in regions with high incidence angles where there are errors in the photometrically corrected mosaic. It is possible that a small number of these dark faculae, which were not spatially extensive enough to be detected with VIR, are not compositionally similar to the carbonate-bearing faculae and may instead be impactor material from bright asteroids.

We group faculae into four geologic settings (Fig. 2): (1) central pit or peak complexes or floor fractures within large craters ("floor faculae"), (2) crater rims and walls ("rim/wall" faculae), (3) 


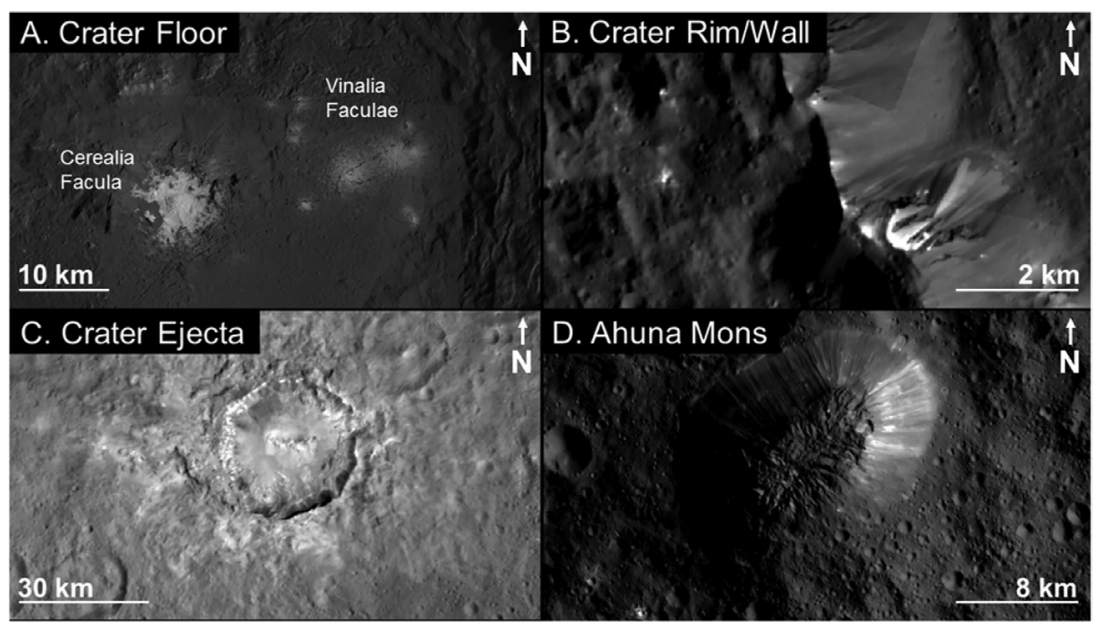

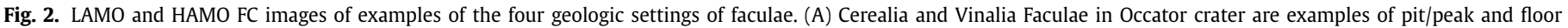

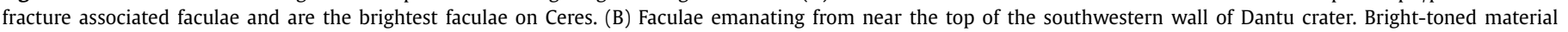

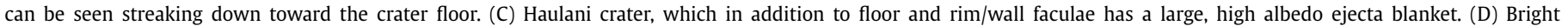
material streaks down the steep slopes of the mountain Ahuna Mons. North is up in all images.

Table 1

List of all craters with floor faculae, their dimensions, and presence/type of floor fractures (Buczkowski et al., 2016) and pitted terrains (Sizemore et al., 2017).

\begin{tabular}{|c|c|c|c|c|c|c|}
\hline Crater name & Diameter $(\mathrm{km})$ & Approx. depth (m) & Approx. depth diameter ratio & Occurrence & Floor fractures? & Pits? \\
\hline Occator & 92 & 4200 & 0.046 & Central pit/dome and floor fractures & Class 1 & Few \\
\hline Dantu & 125 & 5180 & 0.041 & Floor fractures & Class 1 & Abundant \\
\hline Haulani & 32 & 3050 & 0.095 & Central peak & Class 1 & Abundant \\
\hline Ikapati & 50 & 3560 & 0.071 & Central peak complex & Class 1 & Abundant \\
\hline Gaue & 89 & 4430 & 0.050 & Central peak & Class 1 & No \\
\hline Azacca & 50 & 3980 & 0.080 & Central peak complex & Class 1 & Few, degraded \\
\hline Urvara & 163 & 4500 & 0.028 & Central peak & Possible class 4 & Degraded \\
\hline Ernutet & 57 & 3250 & 0.057 & Central peak & No & No \\
\hline
\end{tabular}

within ejecta, including the rims or walls of small craters in ejecta blankets ("bright ejecta"), and (4) the mountain Ahuna Mons. With the exception of Ahuna Mons, all faculae occur in or around impact craters (Fig. 3). By latitude, the faculae are relatively evenly distributed within the $\pm 55^{\circ}$ latitude bounds of our study area and are prevalent throughout both Vendimia Planitia and Hanami Planum. There is no clear correlation between facula concentration and crustal thickness, although the extensive Cerealia Facula is located in the thickest portion of the crust (Ermakov et al., 2017). Faculae appear associated with many, but not all (see Sections 3.1.1 and 3.2.1), of the least-degraded craters and with some older basins. In this manuscript, we focus on the characteristics of faculae-containing craters, i.e., settings 1-3 above.

\subsection{Floor faculae}

\subsubsection{Characteristics of craters with floor faculae}

Floor faculae are defined as faculae that occur within the central pit or peak complex of large craters or along crater floor fractures (Fig. 4), and are distinct from faculae exposed on the rim or wall of small craters contained within the floor of larger craters (e.g. in Fig. 4E and F). Floor faculae are observed in eight craters: Occator, Dantu, Haulani, Azacca, Ikapati, Gaue, Ernutet, and Urvara, and range in extent from $<1 \mathrm{~km}^{2}$ (Gaue) to $>120 \mathrm{~km}^{2}$ (Occator) (Table 1). Cumulatively these faculae span a total area of at least $375 \mathrm{~km}^{2}$. Each of these craters is larger than the simple-tocomplex crater transition diameter, which on Ceres occurs at diameters of 7.5-12 km (Hiesinger et al., 2016). The smallest of the craters exhibiting floor faculae is Haulani, with a diameter $D$ of $\sim 32 \mathrm{~km}$, and the largest is Urvara with $D \sim 163 \mathrm{~km}$. Most large craters, including more than $90 \%$ of craters with $D>50 \mathrm{~km}$ and the largest crater on the surface (Kerwan, $D=283 \mathrm{~km}$ ), do not con- tain floor faculae (Fig. 5A). An additional important parameter is crater depth, independent of diameter. Craters with floor faculae are among the deepest on the surface; 8 of the 28 deepest craters contain floor faculae, and 13 of the 28 deepest craters contain at least some type of bright material. Although the craters with floor faculae are not anomalously deep for their diameter relative to the average crater population, they are the deepest in their respective regions (Fig. 5B) with only one exception, Ernutet, which is adjacent to the deeper Ikapati crater, which also has floor faculae. Hence, crater depth is a stronger control on the presence of floor faculae than crater diameter alone. No craters shallower than $3 \mathrm{~km}$ contain floor faculae. No correlation exists between crater diameter or depth and the spatial extent of floor faculae.

Most of the floor facula-containing craters exhibit features consistent with the upwelling and outgassing of volatiles. Urvara, Dantu, Occator, Ikapati, Azacca, and Haulani contain pitted terrains on their floors consistent with post-impact outgassing of volatiles (Denevi et al., 2012; Sizemore et al., 2017). Dantu and Ikapati contain the largest examples of pitted terrains on the surface. Occator, Azacca, Gaue, Dantu, Haulani, and Ikapati contain fractures on their floor that are morphologically similar to class I lunar FloorFractured Craters (FFCs), whose formation is attributed to doming resulting from magmatic intrusion (Schultz, 1976; Jozwiak et al., 2012; Buczkowski et al., 2016).

The craters with floor faculae generally exhibit morphologically immature features including steep crater sides and welldefined central peak/pit complexes. Crater size-frequency distribution (CSFD) measurements indicate that the craters with floor faculae are relatively young. ADM crater ages range from $<6 \mathrm{Ma}$ for Haulani to $110 \pm 7.2 \mathrm{Ma}$ for Urvara and $420 \pm 60 \mathrm{Ma}$ for Ernutet, the two oldest craters with floor faculae (Schmedemann et al., 2016; Pasckert et al., 2017). FC color images show that the floor 

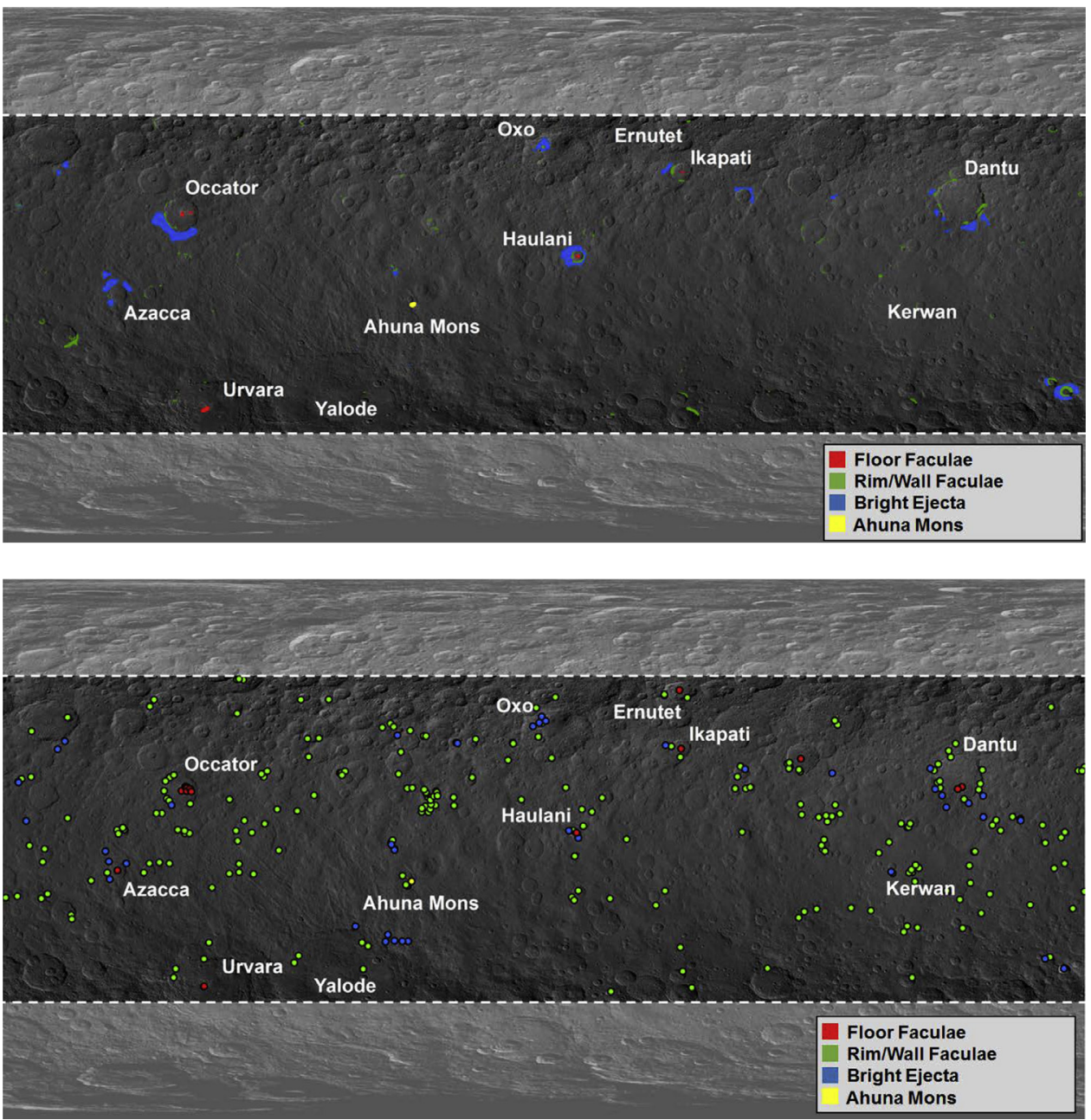

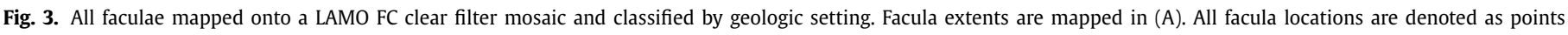
in $(B)$.

facula craters occur in spectrally blue regions, which have a negative spectral slope over the visible wavelengths relative to the average surface, consistent with their young age (Jaumann et al., 2016; Nathues et al., 2016; Schmedemann et al., 2016; Stephan et al., 2017). Floor formation ages of the floor facula craters are known in some cases to be significantly younger than crater formation ages, indicating extensive and sometimes long lasting postimpact activity (Nathues et al., 2016).

\subsubsection{Characteristics of floor faculae}

The floor faculae are typically, though not exclusively, associated with central pit or peak complexes (Fig. 4A-F and $\mathrm{H}$ ) and exhibit an array of morphologies. The brightest and most extensive is Cerealia Facula in the $\sim 3.5 \mathrm{~km}$ wide, $0.6 \mathrm{~km}$ deep central pit of Occator crater (Jaumann et al., 2017; Nathues et al., 2017a). A dome in the center of the pit consisting of bright material rises $0.4 \mathrm{~km}$ above the surrounding terrain and exhibits extensional tectonic fractures (Nathues et al., 2017b). The average Bond albedo of Cerealia Facula is $0.24 \pm 0.01$ and increases to more than 0.5 in the center of the dome. Small craters in Cerealia Facula appear to expose brighter underlying material (Fig. 4A). A cluster of several faculae (Vinalia Faculae) lies on the eastern floor of Occator along an extensive fracture system (Nathues et al., 2017a). These faculae are on average significantly darker than Cerealia Facula, with a Bond albedo of $0.11 \pm 0.04$.
In Haulani crater, faculae with an albedo of $0.085 \pm 0.01$ are exposed near the top of a central mountain ridge and streak downslope alongside smooth, lobate landslide materials that are mass wasting toward the crater floor (Fig. 4B). The crater floor contains numerous pits, flows, and floor fractures as well as diffuse bright material. Extensive faculae are also exposed along the rim. Faculae in Azacca, Gaue, Urvara, and Ikapati also occur along central ridges or peak complexes (Fig. 4C-F), but are not directly associated with lobate flows as in Haulani. Gaue, Urvara, and Ikapati also contain smooth floor materials that may be sourced from post-impact extrusion of volatile-rich material (Pasckert et al., 2016; Sizemore et al., 2017). In Dantu crater, faculae with an average albedo of $0.11 \pm 0.02$ occur along crater floor fractures rather than in the central pit/peak complex and are the most spatially extensive floor faculae outside of Occator crater (Fig. 4G). The faculae in Ernutet (Fig. 4H) and Gaue (Fig. 4D) are less extensive than those in other craters and although they occur in central peak complexes, they may be associated with small impact craters below the limit of FC resolution, and hence may have formed by a process distinct from the other floor faculae.

We found no clear correlation between crater formation age and albedo of floor faculae; the central facula of Haulani (albedo $0.085 \pm 0.01$ ), the youngest of the craters $(<6 \mathrm{Ma}$, Schmedemann et al., 2016) with floor faculae, is significantly darker than Occator's Cerealia and Vinalia Faculae (albedos of $0.24 \pm 0.01$ and $0.11 \pm 0.04$, respectively). Crater count-based formation age esti- 


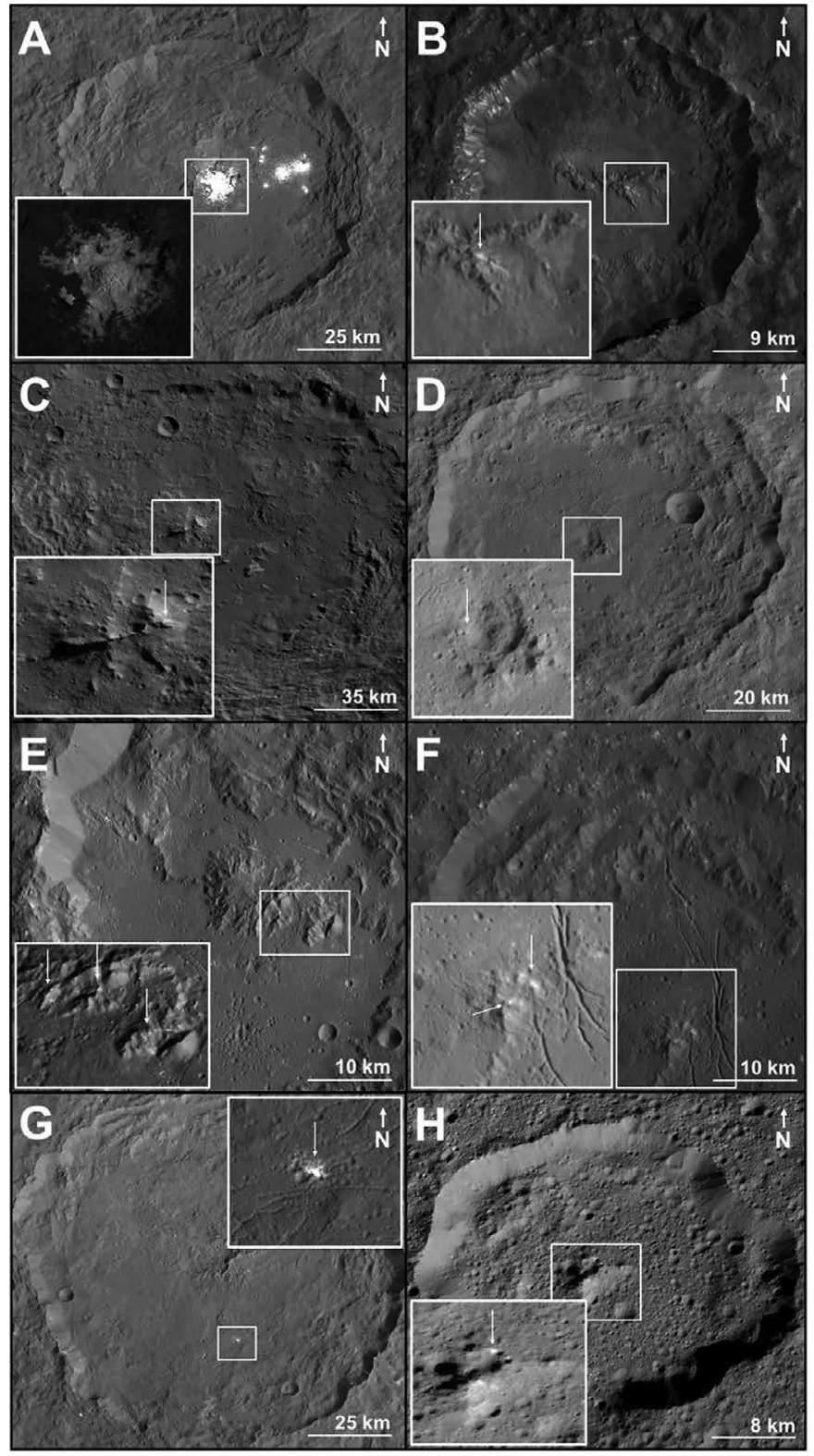

Fig. 4. FC images of all craters with floor faculae. (A) Occator crater with an inset of Cerealia facula in Occator's central pit. Extensional fractures are visible on the $0.4 \mathrm{~km}$ tall central dome (Nathues et al., 2017a). Small impact craters on this facula expose brighter underlying material. (B) Haulani crater. Bright material emanates from near the top of the central ridge alongside a lobate flow. Rim/wall faculae are also visible on the crater's western rim of the crater. (C) Urvara crater, which contains a large facula on its central ridge. (D) Gaue crater, which contains sparse and fairly dark faculae near its central pit. (E) Faculae in the central peak complex of Ikapati crater. Rim/wall faculae are also visible in small craters on the crater floor and along the western crater rim. (F) Faculae in the central peak complex of Azacca crater. Rim/wall faculae are also visible in small craters on the crater floor and along the western crater rim. $(G)$ Dantu crater, which contains a large floor facula associated with concentric floor fractures. $(\mathrm{H})$ Faculae on the central ridge of Ernutet crater. Rim/wall faculae are also visible on the western and southern crater rims.

mates for Occator crater are $34 \pm 2 \mathrm{Ma}$ (Nathues et al., 2017b) and $23.7 \pm 1.1 \mathrm{Ma}$ (Neesemann et al., This issue). The Haulani floor facula is also darker than the floor faculae in the 72-150 Ma Dantu crater (albedo $0.11 \pm 0.02$ ), but brighter than the faculae in the older Azacca crater (albedo $0.07 \pm 0.01$ ) (Fig. 6).
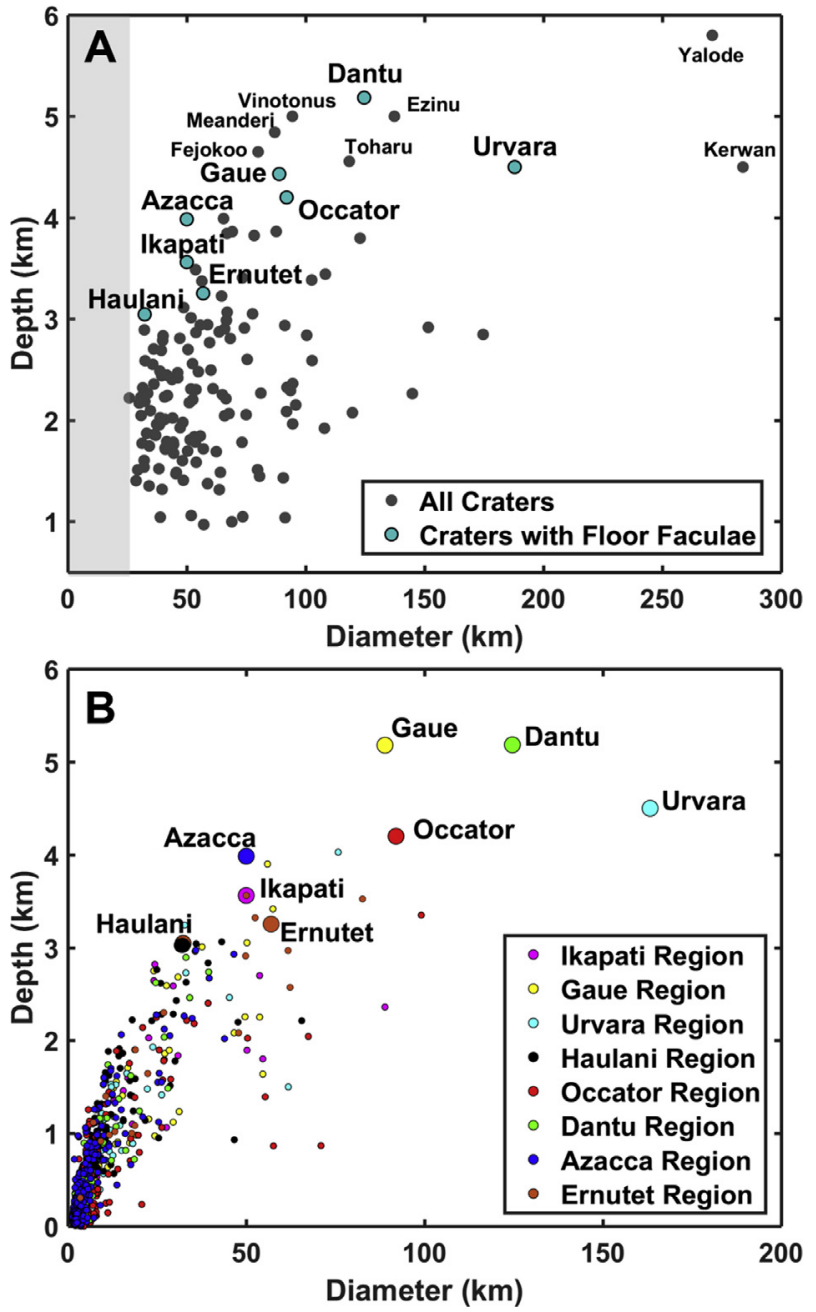

Fig. 5. (A) Depth and diameter of the largest craters on Ceres (gray) with those that contain floor faculae indicated (cyan). (B) Depth and diameter of craters within a $\sim 200 \mathrm{~km}$ radius of the craters with floor faculae. A $\sim 200 \mathrm{~km}$ radius was selected under the assumption that the subsurface composition is relatively similar over that area. (For interpretation of the references to color in this figure legend, the reader is referred to the web version of this article.)

\subsection{Rim/wall faculae}

\subsubsection{Characteristics of craters with rim/wall faculae}

Rim/wall faculae and collections of bright material occur along the rims or walls of craters, typically within $\sim 100-200 \mathrm{~m}$ of the uppermost surface (Fig. 2B). By number and extent, they are the most common facula class, occurring in more than 150 craters with $D>1 \mathrm{~km}$ and covering at least $1100 \mathrm{~km}^{2}$ cumulatively. The craters containing rim/wall faculae are often clustered together in small groups. Unlike floor faculae, rim/wall faculae occur in craters of all sizes, including the smallest resolvable craters $(\sim 100 \mathrm{~m}$ diameter) in LAMO FC images, and most frequently occur in simple craters (Fig. 7A). The distribution of craters with rim/wall faculae is, however, skewed toward larger diameters relative to Ceres' average crater population (Fig. 7B). Rim/wall faculae also occur preferentially in craters that are anomalously deep for their diameter (Fig. 7C). The high $d / D$ of craters with rim/wall faculae indicates that on average they are relatively young. Not all fresh craters contain rim/wall faculae, but faculae are not observed in any highly degraded craters.

Some of the most extensive rim/wall faculae occur in craters with floor faculae such as Haulani and Dantu, and all craters 


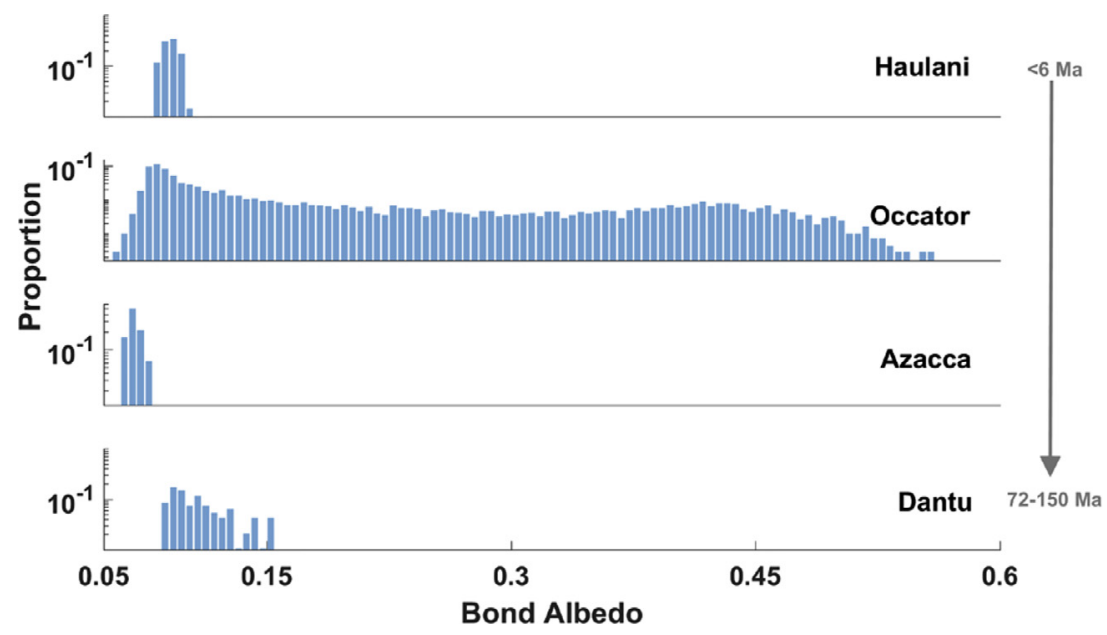

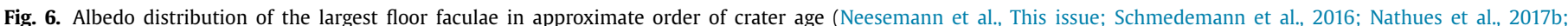

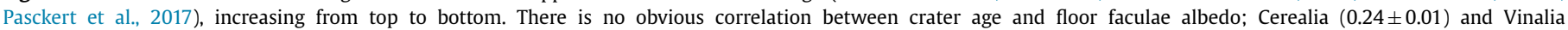

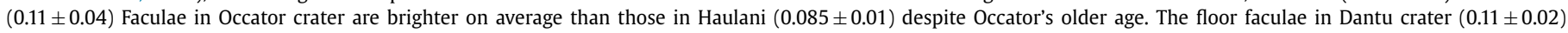
are significantly brighter than those in Azacca $(0.07 \pm 0.01)$, despite Dantu being significantly older.

with floor faculae also contain rim/wall faculae. Additionally, the floors of craters with floor faculae also contain many small craters with rim/wall faculae. Many craters with extensive rim/wall faculae, such as Haulani (Fig. 4B), Kupalo (Fig. 8A), Juling (Fig. 8A), Oxo (Fig. 8B), and Ikapati (Fig. 8C) also contain bright ejecta blankets (see Section 3.3). Some of the brightest and most extensive rim/wall faculae occur in the adjacent and geologically young Juling $(D=20 \mathrm{~km})$ and Kupalo $(D=26 \mathrm{~km})$ craters (Fig. $8 \mathrm{~A})$.

\subsubsection{Characteristics of rim/wall faculae}

Rim/wall faculae typically occur as isolated $<1 \mathrm{~km}$ diameter bright regions that emanate from the shallow subsurface and streak down crater walls. In some large craters, faculae are observed near the base of crater walls or even on crater floors, but closer examination reveals that these actually occur on the rims of smaller, later impacted craters. In some craters such as $\mathrm{Ku}-$ palo (Fig. 8A), faculae are more extensive and occur in discontinuous clusters that line portions of the crater rim at equal depth. The faculae frequently streak down crater walls, darkening with lateral distance down a topographic gradient (Fig. 2B). In smaller craters such as Oxo (Fig. 8B), bright material that lines the entire crater rim as well as portions of the crater floor was likely excavated instead of forming upon impact, as the floor bright material is contiguous with bright ejecta. Most rim/wall faculae occur in small craters within bright ejecta blankets, which likely expose even brighter underlying material (e.g., Fig. 8C).

Rim/wall faculae have an average Bond albedo of $0.10 \pm 0.01$, significantly lower than the average floor facula albedo of $0.18 \pm 0.02$, but this difference in averages is primarily attributable to Occator's spatially extensive and exceptionally bright Cerealia Facula, without which the average floor facula albedo drops to $0.089 \pm 0.01$. Without Oxo, which contains by far the brightest $\mathrm{rim} /$ wall facula, the average rim/wall facula albedo is $0.07 \pm 0.01$. Many rim/wall faculae are brighter than floor faculae in other craters. Within the same crater, however, rim/wall faculae are darker than the floor faculae (Fig. 9). In contrast to the noncorrelation between albedo and crater formation age for floor faculae, there is a negative correlation between rim/wall facula albedo and crater age with younger craters containing brighter faculae on average (Fig. 9). The different trends in albedo between floor and rim/wall faculae are interpreted to result from activity in the floor faculae that potentially significantly post-dates crater forma- tion (see Section 4.1.1), making floor faculae brighter than the parent crater age might suggest.

\subsection{Bright ejecta}

Many craters with floor and/or rim/wall faculae exhibit bright ejecta blankets. These bright ejecta are only associated with craters that also contain rim/wall faculae, and they occur around craters of all sizes. Although there is no preferred overall orientation of the bright ejecta, they are preferentially on the sides of craters where rim/wall faculae are most prominent, such as at Juling and Kupalo (Fig. 8A). Around somewhat older craters such as Dantu and Ikapati, bright ejecta contain within them small craters with $\mathrm{rim} /$ wall faculae that expose brighter underlying material (Fig. 8C). The ejecta are on average darker than rim/wall and floor faculae, perhaps because of partial mixing with average surface material (Fig. 10).

\subsection{Ahuna Mons}

Ahuna Mons is a $\sim 5 \mathrm{~km}$-high, $17 \mathrm{~km}$-wide mountain near Ceres' equator and the most prominent mountain on the surface (Fig. 2D). The summit is concave downward and contains troughs, ridges, and hummocky terrains, while the mountain flanks are at the angle of repose and contain bright material that streaks downward from the summit. On the east face of Ahuna Mons, bright material appears to emanate from the top of the mountain and streak $\sim 4 \mathrm{~km}$ to its base, likely because of gravity-driven motion. On the north flank, similar streaks of bright material appear to emanate from $\sim 1-2 \mathrm{~km}$ above the surface on the side of the mountain and streak to the surface. Several small craters on the south flank and top of Ahuna Mons expose rim/wall faculae. Previous modeling by Ruesch et al. (2016) indicates that Ahuna Mons was emplaced by the formation of a cryovolcanic dome, likely from extrusions of hydrated salts with low eutectic temperatures and thermal conductivities. The bright streaks likely run out due to gravity-driven regolith motion. No similar faculae are observed on any other mountains independent of rim/wall faculae in impact craters.

\subsection{Composition of faculae}

Palomba et al. (2017) and Carrozzo et al. (2017) investigated the composition of faculae using VIR spectra and found significant variations in the abundance of ammoniated clays and the 

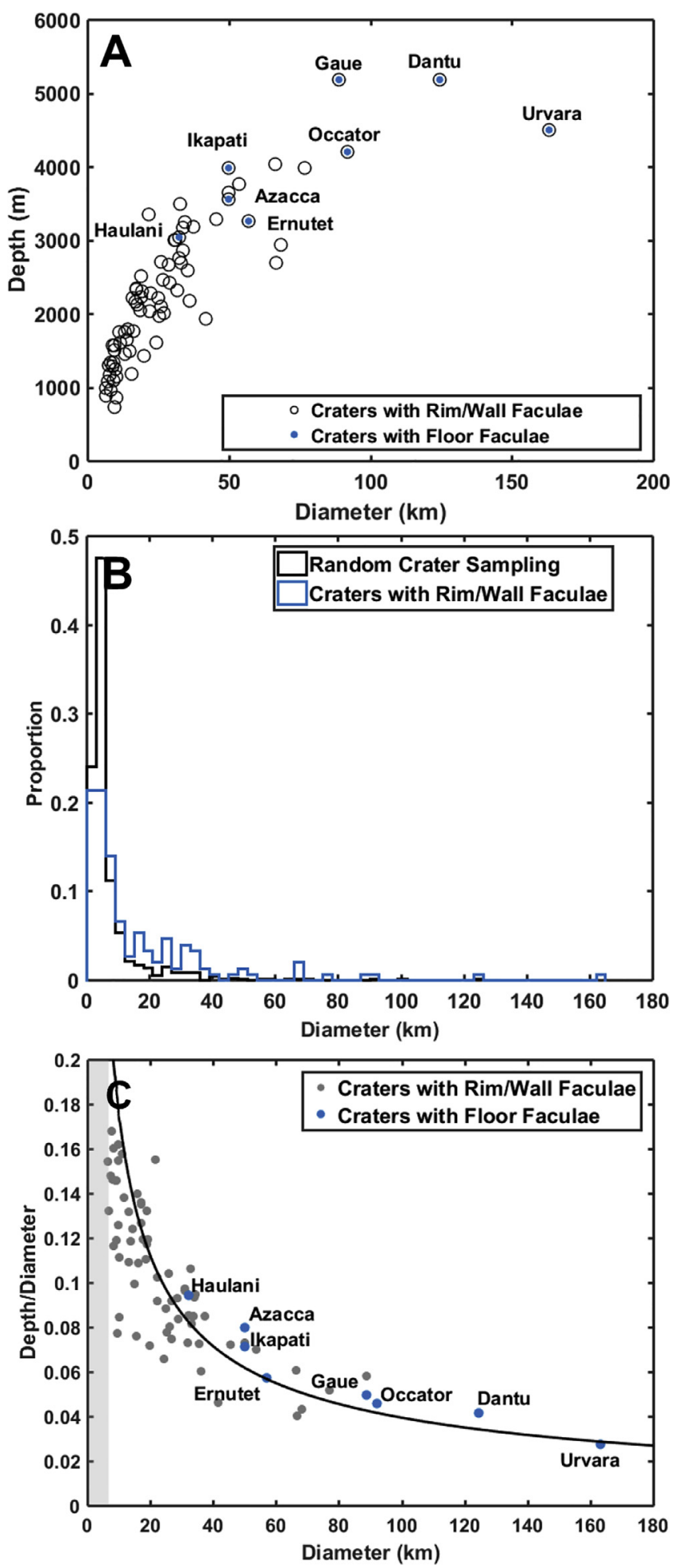

Fig. 7. (A) Depth and diameter of $>5 \mathrm{~km}$ diameter craters that contain floor faculae (blue circles) and rim/wall faculae (black circles). (B) Comparison of the diameter of craters with rim/wall faculae (blue) to a random sample of more than 1000 craters (black) from $\pm 55^{\circ}$ latitude. (C) The depth/diameter of craters with rim/wall faculae (black and blue) as a function of diameter compared with a curve representing the average depth/diameter of morphologically fresh craters on Ceres (black). On average, the depth/diameter of craters with rim/wall faculae is close to that of other morphologically fresh craters, consistent with a young crater age. (For interpretation of the references to color in this figure legend, the reader is referred to the web version of this article.) depth and position of 3.4 and $4 \mu \mathrm{m}$ carbonate absorption bands across the surface. Most faculae contain $\mathrm{Mg}-\mathrm{Ca}$ carbonates similar in composition to Ceres' average surface carbonates, mixed with dark components and hydrated and ammoniated compounds (Palomba et al., 2017). Several regions including Oxo, Occator, Azacca, Kupalo, Ernutet, Dantu, Haulani, Baltae Catenae, and Ahuna Mons contain some Na carbonate associated with bright material (Zambon et al., 2017; Carrozzo et al., 2017). The rim/wall faculae are compositionally similar to many floor faculae, but the dominant carbonate tends to be $\mathrm{Mg}-\mathrm{Ca}$ carbonate whereas floor faculae and the most extensive rim/wall faculae (e.g. in Oxo and Kupalo) typically contain at least some Na-carbonate (Carrozzo et al., 2017; Palomba et al., 2017). Water ice detections in Oxo crater (Combe et al., 2016; Nathues et al., 2017b) and other $\mathrm{H}_{2} \mathrm{O}$-containing craters (Carrozzo et al., 2017; Combe et al., 2017) are sometimes contiguous with the carbonate-bearing faculae.

\section{Potential facula formation mechanisms}

Apart from Ahuna Mons, the formation of faculae is clearly associated with impact events, although the formation mechanisms behind the different types of faculae are likely distinct. The occurrence of floor faculae in exceptionally deep craters is consistent with their formation via (A) impact-induced heating and upwelling of volatile-rich materials, (B) excavation/upwelling of heterogeneously distributed brines or their precipitation products, or (C) a combination of both processes with some variation by location. The occurrence of rim/wall faculae in craters of all sizes cannot be explained by impact-induced heating or upwelling, and hence their formation requires the induction of a separate formation mechanism, likely the excavation of previously emplaced faculae, such as buried floor faculae or exposed brine layers or lenses. Here we evaluate different facula formation mechanisms in the context of the number of faculae and morphological observations discussed in Sections 2 and 3.

\subsection{Floor facula formation}

The formation of floor faculae is clearly associated with impact events, and the formation process must allow for the transport of volatiles from the subsurface to the surface. Below about $40^{\circ}$ latitude in both hemispheres, Ceres' surface is largely devoid of water ice and other volatiles at $\sim 1 \mathrm{~m}$ depth (Prettyman et al., 2016), but the shallow subsurface is likely a mixture of $30-40 \%$ ice by volume with an intermixed silicates, salts, and clathrates (Bland et al., 2016). Previously, De Sanctis et al. (2016) proposed that natrite $\left(\mathrm{Na}_{2} \mathrm{CO}_{3}\right)$, likely the predominant carbonate of many floor faculae, and accompanying ammonium-bearing species could have formed by the interaction of $\mathrm{NaCl}$ and $\mathrm{NH}_{3}$ in solution. The maximum modeled temperature of Ceres' outer crust (Castillo-Rogez and McCord, 2010; Hayne and Aharonson, 2015) is as high as $-30^{\circ} \mathrm{C}$, below the eutectic points of $\mathrm{NaCl}\left(-21^{\circ} \mathrm{C}\right), \mathrm{NH}_{4} \mathrm{Cl}\left(-22^{\circ} \mathrm{C}\right), \mathrm{NH}_{4} \mathrm{HCO}_{3}$ $\left(-17^{\circ} \mathrm{C}\right)$, and $\mathrm{NaHCO}_{3}\left(-6^{\circ} \mathrm{C}\right)$, the likely Ceres salts, in water.

\subsubsection{Facula formation via impact-induced heating}

One potential mechanism for the production of the facula source fluids is impact-induced heating in large craters (De Sanctis et al., 2016). Crater impact models by Bowling et al. (2016) indicate that material near the center of moderate-to-large impact craters could be heated to more than $270 \mathrm{~K}$, in excess of the eutectic temperature of the salts, and that this heated material could be sourced from depths in excess of $20 \mathrm{~km}$ in an Occator-sized crater. Such a formation scenario is outlined in Fig. 11A. In this scenario, Ceres' near-surface is volatile-depleted and consists of a mixture of low-albedo materials, magnesium phyllosilicates, ammoniated phyllosilicates, and carbonates, with no more than a few wt\% $\mathrm{H}_{2} \mathrm{O}$. 


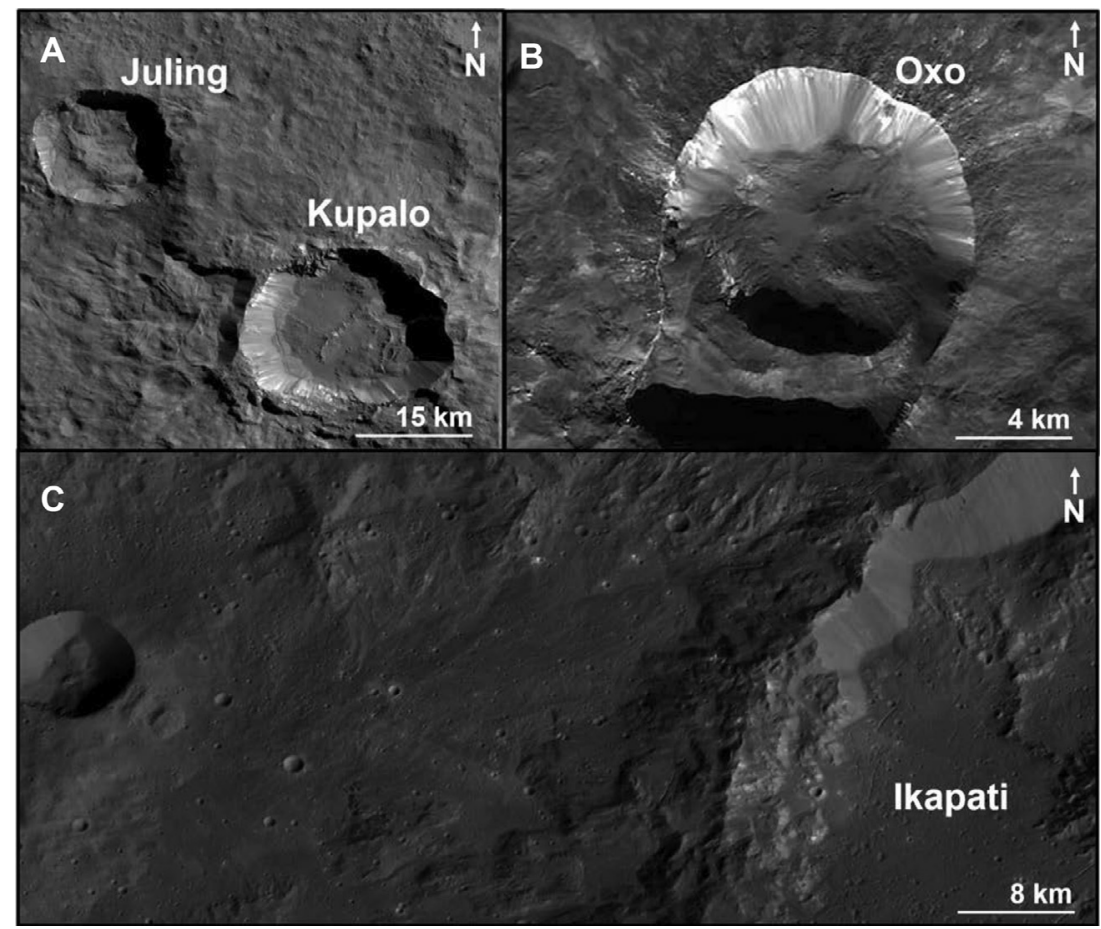

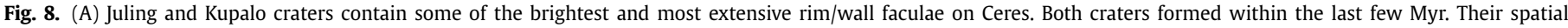

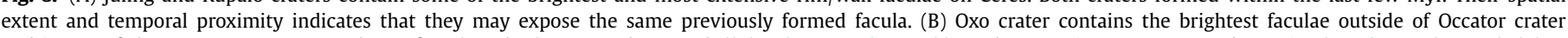

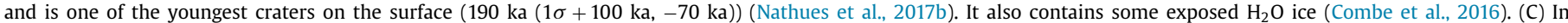

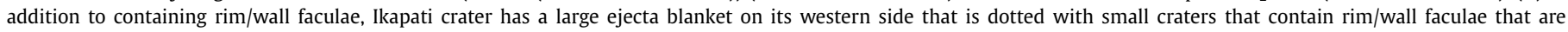
brighter than the surrounding blanket.

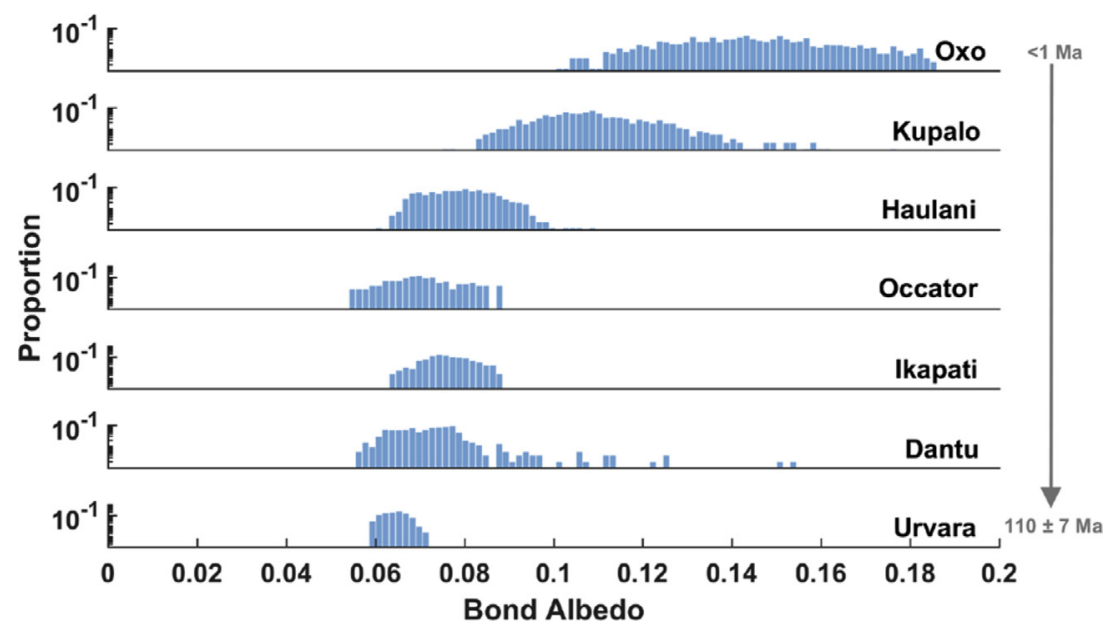

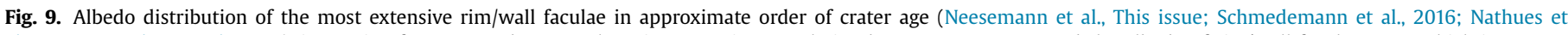

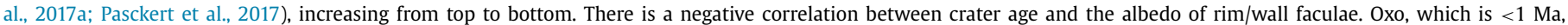
contains the brightest faculae $(0.014 \pm 0.02)$ outside of Occator crater.

The deeper subsurface is enriched in volatiles, especially ice. Heating from a large impact could produce a volatile-rich brine in the subsurface that rises to the surface along impact-induced fractures and pools in a central pit or travels laterally along crater floor or subsurface fractures (Fig. 11Aii). The volatile-rich material would quickly sublimate (Fig. 11Aiii), leaving a bright lag deposit of salts on the crater floor (Fig. 11Aiv).

Models indicate that a heated subsurface region with a characteristic length scale of $10 \mathrm{~km}$ should cool on a timescale of $<3 \mathrm{Ma}$ (Bowling et al., 2016), significantly less than the age of Occator crater, suggesting the formation of faculae could be limited to the period shortly following crater emplacement. It is unclear, however, whether the formation of all floor faculae is contemporane- ous with crater formation; Nathues et al. (2017a) derived a model age of $4.0 \pm 0.1 \mathrm{Myr}$ for Cerealia Facula, significantly younger than Occator itself ( $\sim 20-35 \mathrm{Myr})$. Crater count-based facula ages rely on accurate identification of the spatial extent of already small faculae that may have been larger in the past, and should be treated as an approximate upper age bound. Additionally, the possible detection of a diurnal haze layer above Occator crater (Nathues et al., 2015; Thangjam et al., 2016) may indicate ongoing outgassing activity associated with the faculae, though its presence is debated (Schröder et al., 2017). Moreover, the lack of correlation between floor facula albedo and crater age may also indicate that their formation is not contemporaneous with crater formation as would be expected if faculae darken equally over time due to lateral mixing. 


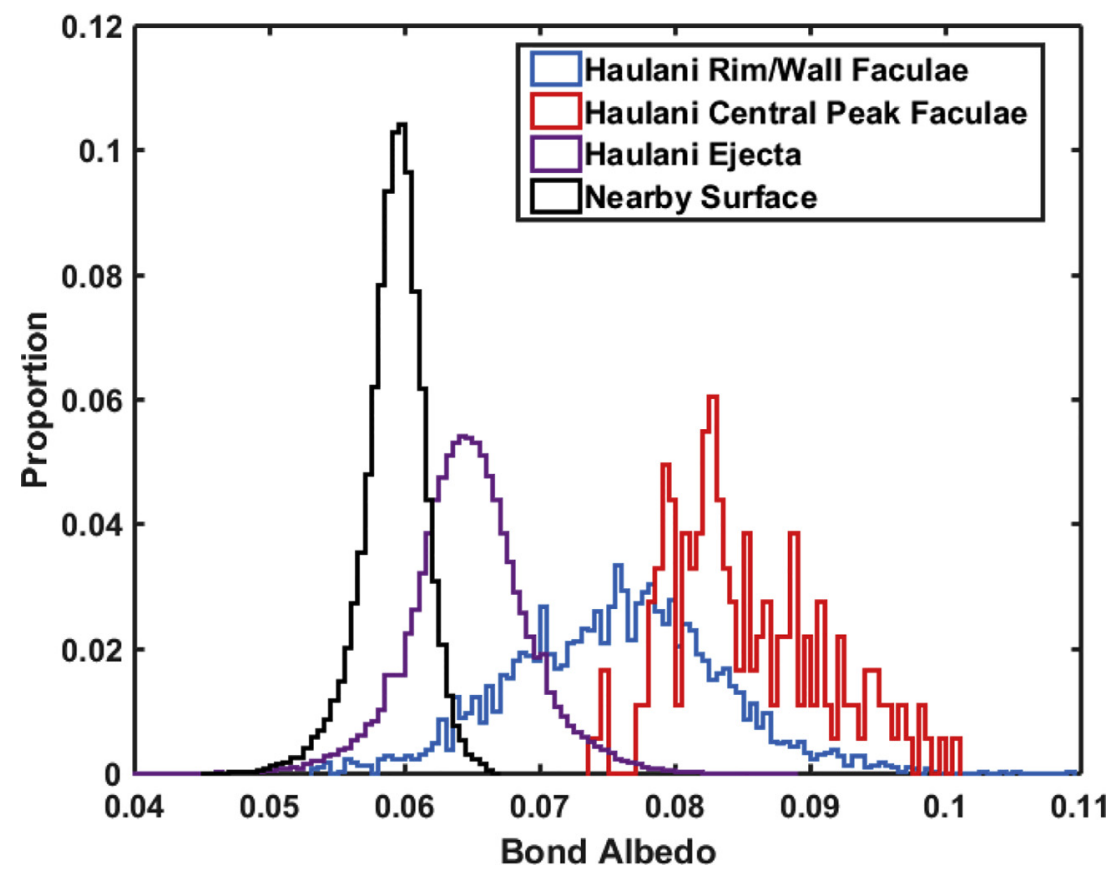

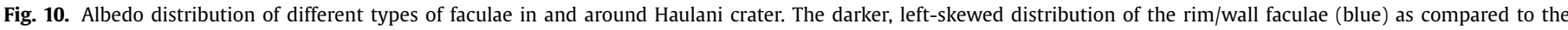

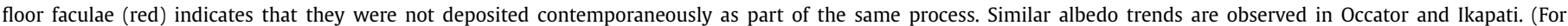
interpretation of the references to color in this figure legend, the reader is referred to the web version of this article.)
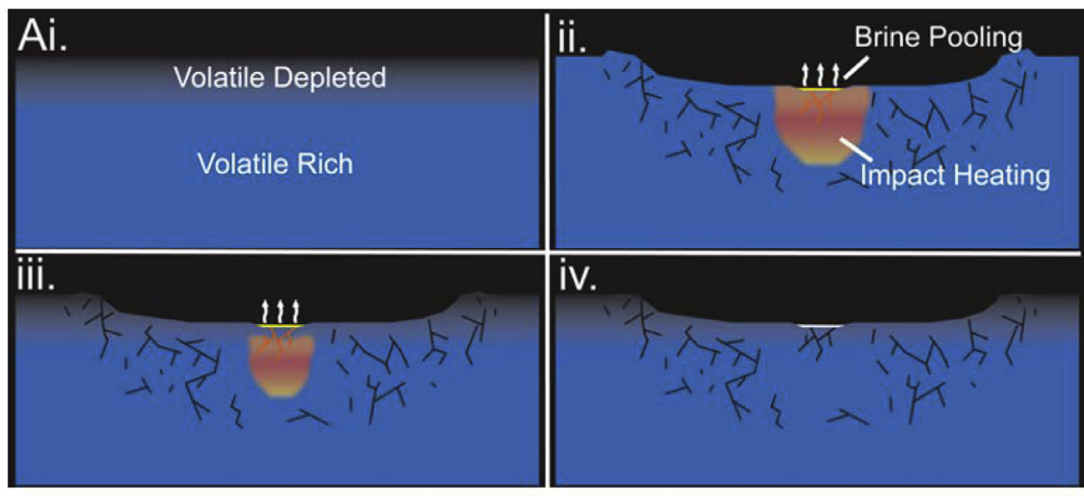

\section{$\mathrm{Bi}$.}
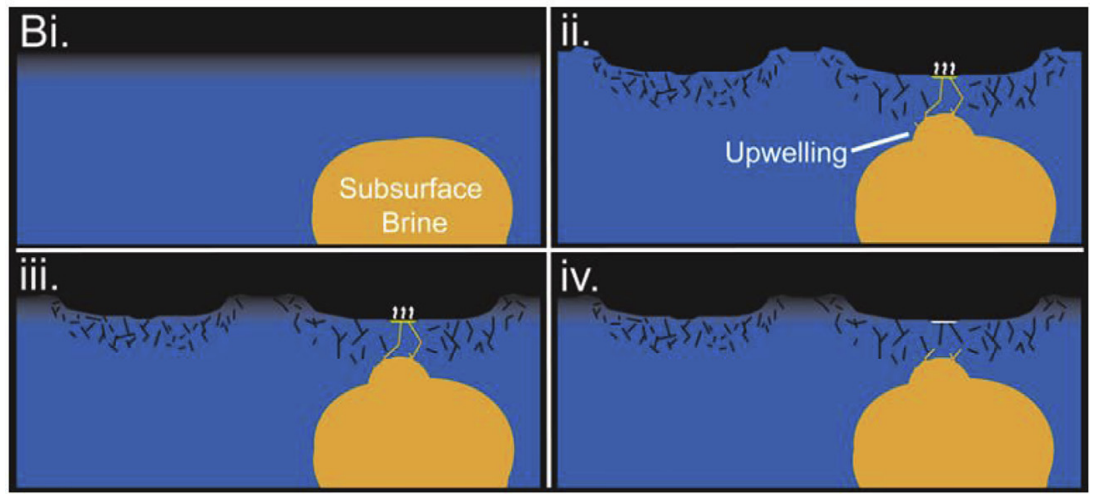

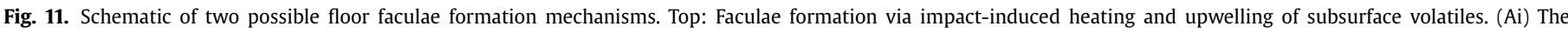

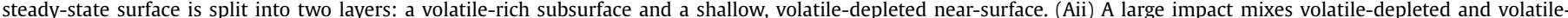

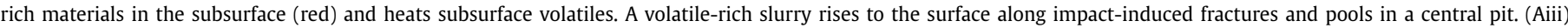

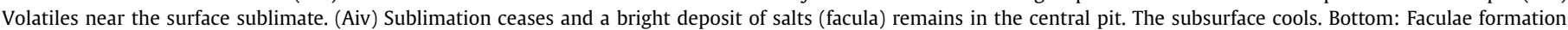

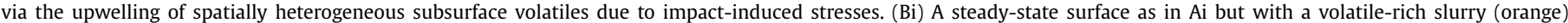

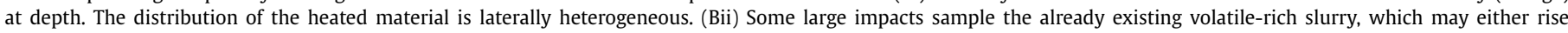

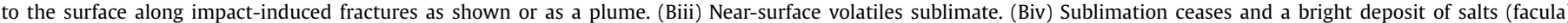

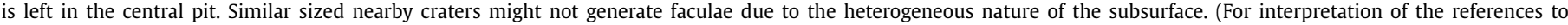
color in this figure legend, the reader is referred to the web version of this article.) 
Hence, the upwelling of material in Occator and other floor facula craters may have persisted for or occurred at least several Myr after impact. Impact heating models must account for the apparent discrepancy between facula and crater ages. Periods of more than $\sim 1$ Myr after impact may be too long if the extrusion of brines is controlled by impact-derived heat alone (Bowling et al., 2016), although upwelling derived from a reservoir of pre-existing volatiles could account for long-lasting post-impact activity.

\subsubsection{Facula formation by upwelling of extant brines from depth}

Cerealia Facula and Ahuna Mons provide a clear indication of recently upwelled subsurface material (De Sanctis et al., 2016; Ruesch et al., 2016; Nathues et al., 2017b). The other floor facula craters also exhibit numerous features consistent with the upwelling of subsurface volatiles such as pitted terrains and floor fractures that are similar to the geometry found in Type 1 lunar floor-fractured craters. Hence, the formation of floor faculae could equally be explained if subsurface temperatures exceed modeled values in at least some locations, allowing volatile-rich brines to exist at depth. Subsequent large impacts could heat these brines further and allow them to travel to the surface along impactinduced fractures (e.g. Fig. 11B) or to rise in plumes as an isostatic response to crater formation. Such a scenario could also allow for upwelling and outgassing activity long after crater formation. Similar processes may have been involved in the formation of Ahuna Mons and its faculae.

In either scenario or a combination of both, the varied compositions of the floor faculae coupled with the lack of floor faculae in several large, deep craters indicates the formation of floor faculae is sensitive to lateral variations in subsurface composition or ice abundance, possibly on the spatial scale of the craters themselves (10s-100s of $\mathrm{km}$ ). Such heterogeneity may be implied by observed variations between the relative abundances of phyllosilicates and ammoniated species (Ammannito et al., 2016). Previous investigations of viscous relaxation by Bland et al. (2016) indicate that Ceres' subsurface ice content may vary by as much as 5-10 vol\% over short length scales. Similar heterogeneities in subsurface temperature or composition could explain the lack of floor faculae in some young, deep craters.

The association of floor facula-containing craters with features consistent with upwelling materials coupled with the young age of Cerealia Facula relative to Occator suggests that the upwelling of extant subsurface brines likely plays an integral role in facula formation and may be complemented by impact-induced heating.

\subsection{Rim/wall facula and bright ejecta formation}

Key observations of rim/wall faculae include darkening with age and their association with impact craters. The occurrence of $\mathrm{rim} /$ wall faculae in many shallow, small-diameter craters is inconsistent with their formation via impact-induced heating or plume activity. Some large craters such as Haulani, Dantu, and Occator contain floor and rim/wall faculae in addition to bright ejecta, which raises the prospect that all types of bright material were emplaced simultaneously by the same mechanism. It is apparent, however, that the rim/wall faculae are darker than floor faculae contained within the same crater, indicating that they were emplaced earlier rather than contemporaneously by the same formation process (e.g., for Haulani, Fig. 10). Bright ejecta blankets have a lower albedo than rim/wall and floor faculae contained in the same crater.

Several observations indicate rim/wall faculae formed via the excavation of previously emplaced faculae: (1) rim/wall faculae occur preferentially in larger diameter craters, as would be expected if new craters were exposing buried bright material because larger craters intersect a greater surface area; (2) craters with rim/wall faculae are frequently clustered together over length scales of 5$50 \mathrm{~km}$, consistent with the existence of heterogeneously distributed subsurface deposits of bright material; (3) all craters with bright ejecta, whose formation is contemporaneous with impact excavation, also contain rim/wall faculae. Previously emplaced floor faculae could have formed via impact-facilitated processes (see Section 4.1) and been later buried by material emplaced by subsequent impacts or subsurface faculae may have already existed at depth from subsurface reactions of volatile-rich brines (Fig. 12A). Subsequent impacts of any size that intersect previously emplaced faculae would expose them again along their crater rim and partially disseminate the material as bright ejecta (Fig. 12B). The association of bright ejecta blankets with rim/wall facula-containing craters, as well as the preferential distribution of bright ejecta on crater sides with rim/wall faculae, indicates that the material comprising these two types of faculae is generated simultaneously by the same mechanism. Subsequently, rim/wall faculae would darken, likely due to space weathering and/or lateral mixing (see Section 5) as indicated by the negative correlation between crater age and rim/wall facula albedo. Eventually the rim/wall faculae are likely buried due to lateral mixing from subsequent impacts (Fig. 12C). Due to the heterogeneous distribution of previously emplaced faculae, not all new impacts would produce rim/wall faculae or bright ejecta.

In the following sections, we examine key aspects of this hypothesized scenario: the timescales for darkening and removal of faculae from the Ceres surface (Section 5) and whether the current number of craters with rim/wall faculae is consistent with their formation via excavation using a simple impact cratering model (Section 6).

\section{Darkening and disappearance of faculae}

The occurrence of floor faculae in exceptionally deep, morphologically fresh craters located in spectrally blue regions indicates that they are geologically young. Recent CSFD measurements by Schmedemann et al. (2016) estimate that the oldest of the floor facula craters has a formation age of $\leq 420$ Ma. The presence of numerous older, shallower $D>50 \mathrm{~km}$ craters (Fig. 4), which may have viscously relaxed to their present state (Bland et al., 2016), and which lack faculae, raises the prospect that at least some of these craters once contained floor faculae that subsequently disappeared from the surface. A similar inference can be drawn for rim/wall faculae, which occur preferentially in anomalously deep and presumably young craters. The lack of rim/wall faculae in degraded craters indicates that either the faculae are a geologically recent phenomenon or that they disappear from the surface over time scales of several hundred Myr. Additionally, a gradual darkening mechanism for the rim/wall faculae is implied by the negative correlation between rim/wall facula albedo and parent crater age. The lack of such a correlation for the floor faculae is interpreted to result solely from the mismatch between floor faculae age and parent crater age due to post-impact activity and is not interpreted to result from a difference in the darkening process.

Possible mechanisms for the disappearance of faculae include gradual devolatilization or chemical alteration, darkening and thus disappearance due to space weathering, or burial via impactinduced lateral mixing. Chemical alteration is unlikely as the known bright constituents of faculae (carbonates and $\mathrm{NH}_{4}$-salts) are stable at the temperatures and pressures of Ceres' surface. Fresh craters on Ceres are spectrally blue and exhibit distinct optical properties compared to older surface regions, indicating that some process of space weathering or regolith gardening homogenizes the surface (Pieters et al., 2016). A different group of surface and space weathering processes including lateral mixing, micrometeorite bombardment and contamination, thermal cycling, and sub- 


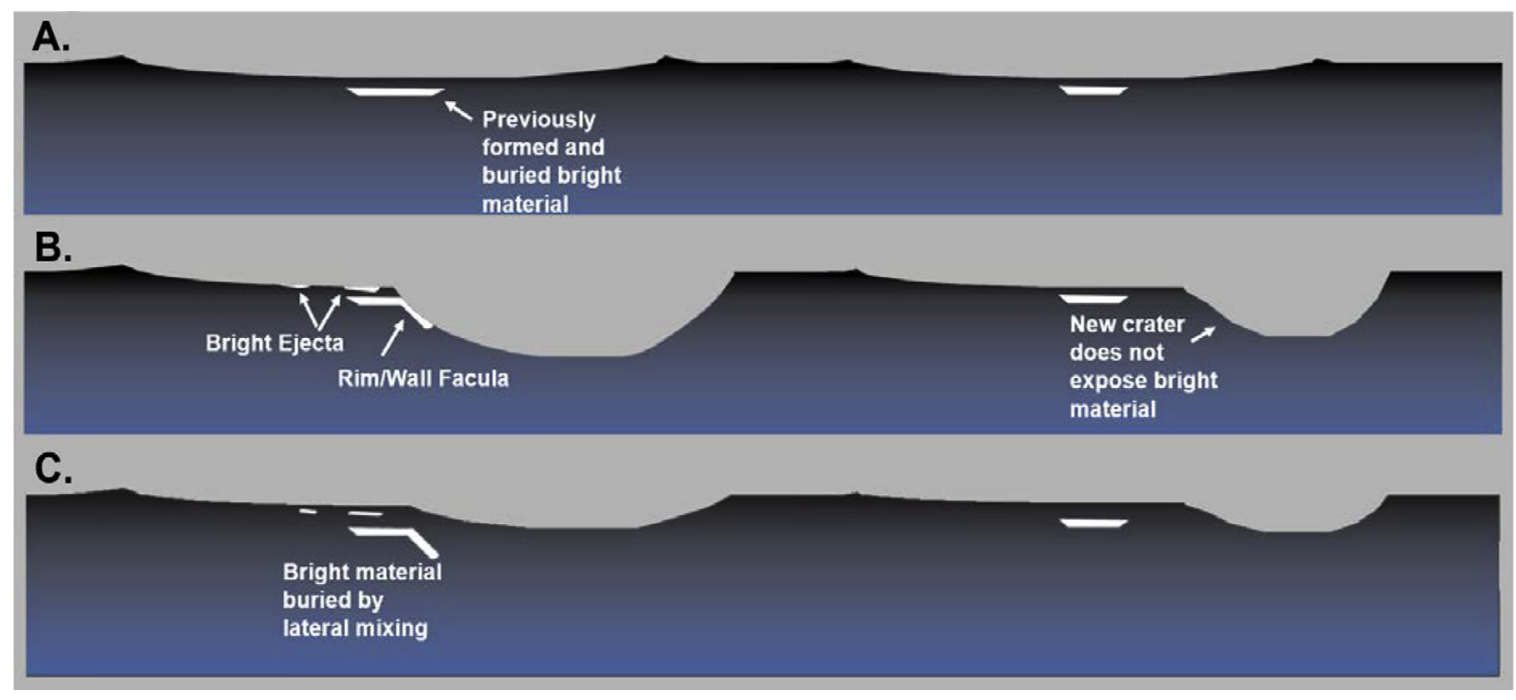

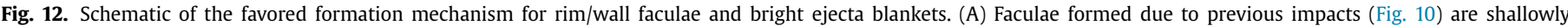

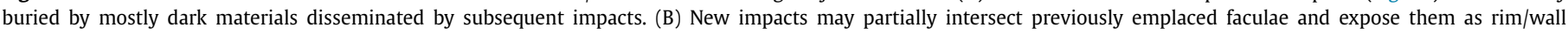

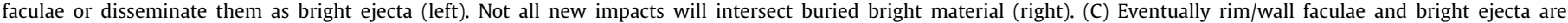
buried due to a combination of space weathering and burial by lateral mixing.

limation likely dominate on Ceres as compared to inner solar system bodies that experience the formation of nanophase metallic iron $\left(\mathrm{npFe}^{0}\right)$ or other nanophase particles (Pieters and Noble, 2016). Of these processes, lateral mixing by emplacement of typical Ceres surface materials is most likely to significantly lower the albedo of faculae over relevant timescales.

We estimated the timescale over which impact-induced lateral mixing and direct impacts are expected to bury or disseminate newly emplaced faculae by using the Ceres crater production function (Hiesinger et al., 2016). A grid with the surface area of Ceres was populated randomly with craters of diameters and frequencies sourced from the crater production function in million year time steps. During each time step the grid was populated with a new set of craters. Ejecta thrown by new craters were assumed to completely bury surface material within a characteristic radius, taken here to be between zero and two crater diameters across. The area covered directly by new craters or their ejecta, which represents a region within which faculae would be removed, was calculated during each time step.

Models were run after $3 \mathrm{Ga}$, after any enhanced early bombardment and over a time period where the cratering production function was similar to the modern production rate. Results show that in the limit of no lateral mixing - i.e. all ejecta are lost to space and faculae are only directly disseminated by impacts - $50 \%$ of faculae would be removed from the surface within $\sim 1.25 \mathrm{Ga}$ (Fig. 13). This represents an upper bound on the average facula removal time from lateral mixing alone. In a more reasonable scenario where ejecta bury surface material within one crater radius, this time decreases to only $\sim 300 \mathrm{Ma}$. The facula removal time would decrease further if the burial radius increased or if other processes like space weathering play a role in removal or darkening of the faculae. Hence, the burial or direct dissemination of faculae by impacts provides a viable mechanism for their removal from the surface over timescales of several hundred Ma, consistent with the observed age range of the floor facula craters.

\section{Facula formation and excavation model}

Under the hypothesis that impacts create crater floor or nearsubsurface faculae with destruction timescales like those above, we next calculated the expected number of faculae produced over Ceres' geologic history and exposed at the surface today. Starting

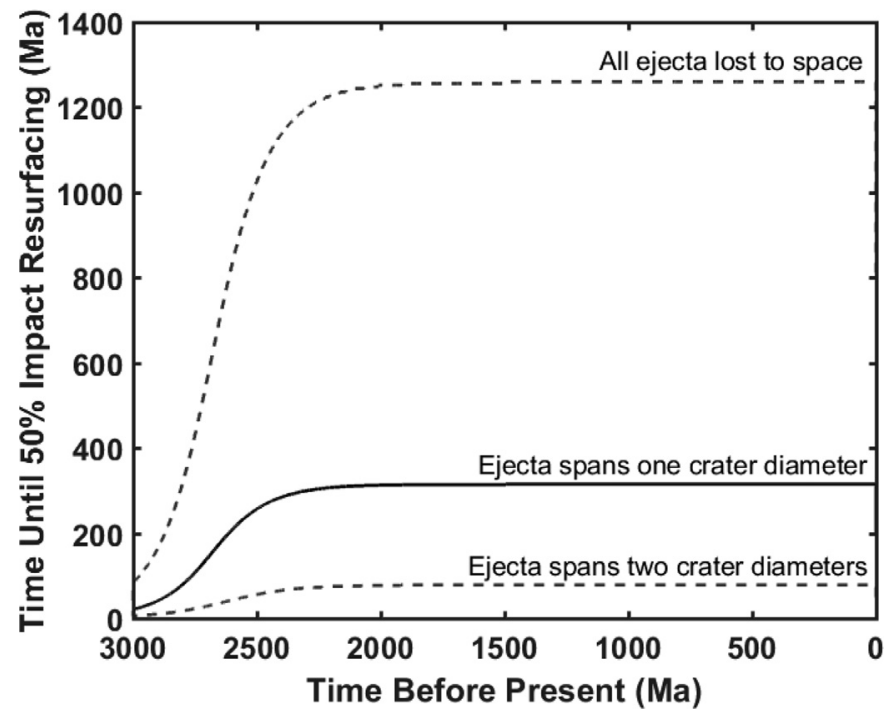

Fig. 13. Simulated time until a given point on Ceres' surface has a $50 \%$ probability of being either directly impacted or buried by impact ejecta at different time periods before the present. The central line assumes that all ejecta are emplaced within a single crater radius. The upper bound denotes the case in which there is not ejecta blanket (i.e. all material is lost to space). The lower bound denotes the case in which the diameter of the ejecta blanket is twice that of the crater. The removal time decreases in the past due to the increased impact rate.

at $t=4.5 \mathrm{Ga}$, a grid with the surface area of Ceres $A$ was populated in Myr time steps with a crater population sourced from the Ceres crater production function $f$ (Hiesinger et al., 2016). During each time step $t_{i}$ a total of $A \sum_{d=1}^{250} f\left(t_{i}, D\right)$ craters were placed at random positions on the grid. Several rules were applied:

- Craters above a cutoff diameter $D_{c}$ produced a circular floor facula of diameter $D_{f}$ in the crater center that was carried to the next time step.

- In subsequent time steps if a new crater completely overlaid a previously emplaced facula, the facula was disseminated as bright ejecta and removed from subsequent time steps.

- If a new crater only partially intersected or was contained completely within a previously emplaced facula, a rim/wall facula was created and carried to the next time step. The portion 

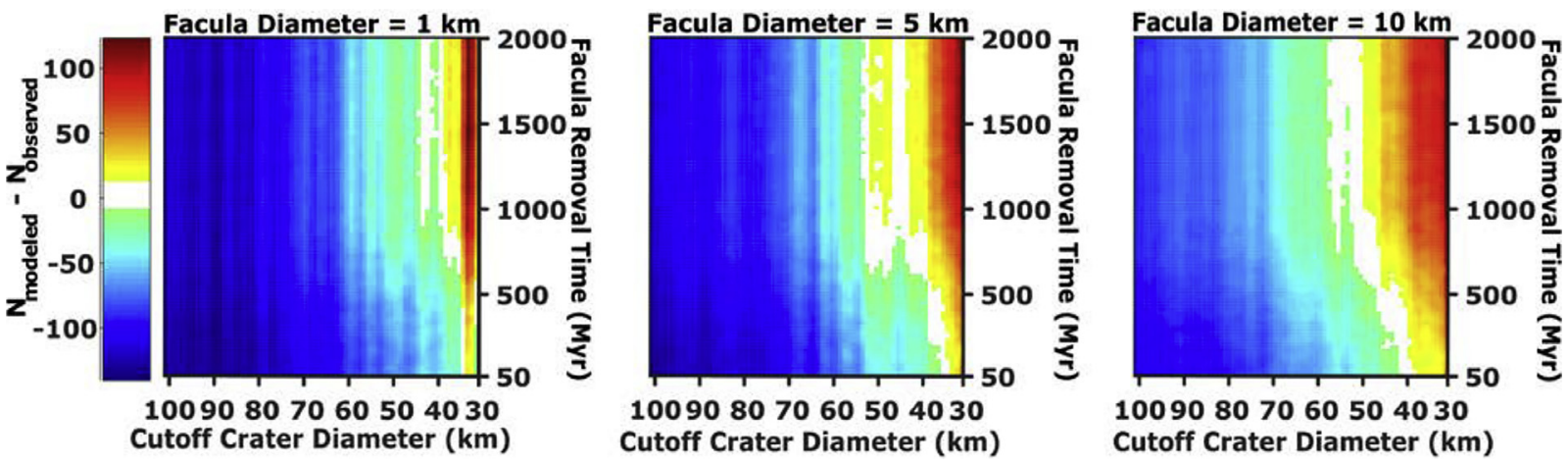

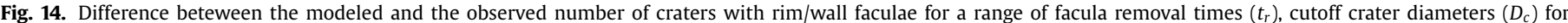

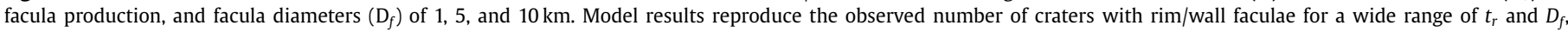
if $D_{c}$ is between 30 and $100 \mathrm{~km}$.

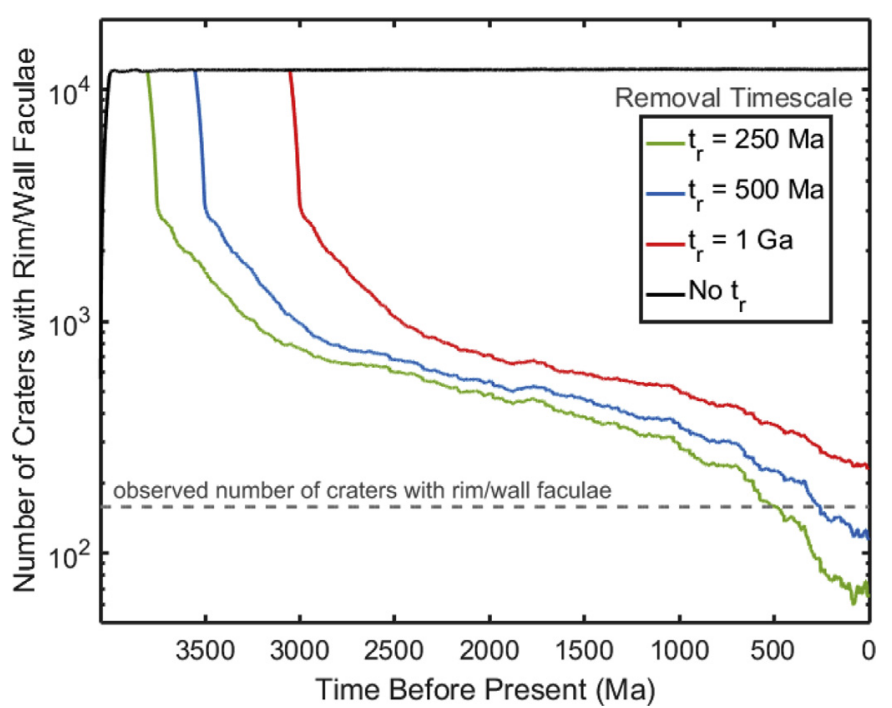

Fig. 15. Simulated number of craters with rim/wall faculae as a function of time for a cutoff crater diameter for faculae production, $D_{c}=75 \mathrm{~km}$, and initial faculae diameter $D_{f}=10 \mathrm{~km}$. The top black line denotes the number of craters with rim/wall faculae if no cutoff time is imposed for facula removal by lateral mixing of dark material ( $t_{r}=0$; i.e. faculae are only removed via direct impacts). The colored lines denote the modeled number of craters with rim/wall faculae if new faculae are removed on a characteristic timescale to simulate the combined effects of darkening due to space weathering and/or burial via lateral mixing. The dashed line denotes the number of craters currently observed with rim/wall faculae independent of ejecta blankets ( 154 craters). (For interpretation of the references to color in this figure legend, the reader is referred to the web version of this article.)

of the previously emplaced floor facula directly intersected by the new crater was disseminated as bright ejecta and removed from subsequent time steps.

- If a new crater completely overlaid a previously emplaced $\mathrm{rim} /$ wall facula, the rim/wall facula was disseminated as bright ejecta, and the rim/wall facula was removed from the simulation.

In addition to destruction by direct impact, the gradual disappearance of faculae was modeled by including a condition whereby any rim/wall faculae remaining after time $t_{r}$ were removed from the surface. The simulation was run for each combination of parameters $D_{c}(50-100 \mathrm{~km}), D_{f}(1-10 \mathrm{~km})$, and $t_{r}(50-2000 \mathrm{Myr})$. The range of $D_{f}$ was selected to mirror the size of faculae observed on the surface, and the range of $t_{r}$ was selected to incorporate the timescales calculated in Section 5. The range of crater diameters $D_{c}$ was selected because larger craters produce floor faculae more frequently than smaller craters, although it is likely unrealistic for every crater with $D>D_{c}$ to produce a facula, as many large and deep craters do not contain faculae. The small number of craters with floor faculae makes this parameter difficult to estimate accurately.

Results show that the model reproduced the currently observed number of craters with rim/wall faculae for a wide range of $D_{f}$ and $t_{r}$ input parameters and a more restricted range of $D_{c}$ (Fig. 14). If no $t_{r}$ is imposed, the modeled number of craters with rim/wall faculae greatly exceeds the $<200$ currently observed. For example, for $D_{c}=75 \mathrm{~km}, D_{f}=10 \mathrm{~km}$, and no $t_{r}$, the final number of craters with $\mathrm{rim} /$ wall faculae exceeds $10^{4}$ (Fig. 15). For the same combination of parameters and $t_{r}$ of 250,500 , and $1000 \mathrm{Myr}$, the simulated number of craters with rim/wall faculae drops to $\sim 50,100$, and 300 , respectively, similar to the observed number. This indicates that the observed number of craters with rim/wall faculae can be explained by the excavation of previously emplaced faculae if they are created by large impacts, distributed by smaller impacts, and removed from the surface over timescales of $<1 \mathrm{Gyr}$. Results also show that there were likely more faculae in the past due to the higher impact rate.

\section{Conclusions}

Images from the Dawn Framing Camera were used to classify, identify, and map all faculae on the surface of Ceres. Faculae were found to fall into four distinct geological classes: (1) crater pits, peaks, or floor fractures (floor faculae), (2) crater rims or walls (rim/wall), (3) bright ejecta blankets, and (4) the mountain Ahuna Mons. Faculae occur almost exclusively in or around impact craters. Floor faculae occur in large, deep, and young craters that often contain crater floor fractures and pitted terrains, consistent with the upwelling and degassing of volatiles. The geometry of craters with floor faculae is consistent with their formation via the impact-induced heating and upwelling of subsurface volatiles, the upwelling of volatile-rich brines along impact-induced fractures or as low-density plumes, or some combination of these processes. The association of floor faculae craters with features consistent with upwelling materials and the young age of floor faculae relative to their parent craters indicate that the impact-initiated upwelling of extant subsurface brines may be the favored crater floor faculae formation mechanism. Variations in the composition and albedo of floor faculae, coupled with the abundance of large and deep craters without floor faculae, indicates that their formation may be dependent on lateral variations in subsurface ice and/or brine abundance, composition, and temperature that only allow faculae to form in some regions of Ceres.

Rim/wall faculae were found to occur in relatively young craters of all sizes, and the geometry of rim/wall faculae and the craters that contain them is consistent with their formation via the excavation of faculae that were previously emplaced either as floor 
faculae that were subsequently buried or by the reaction of subsurface brines. Bright ejecta are interpreted to form by the same mechanism on the basis of their strong association with rim/wall faculae. Models using the Ceres crater production function indicate that faculae are likely to be destroyed, buried by impact-induced lateral mixing, or directly disseminated by impacts on timescales of less than several hundred Myr, consistent with the young age of the rim/wall- and floor facula-containing craters. Space weathering also may play an active role in the speeding the darkening of faculae over Myr timescales. A $4.5 \mathrm{Gyr}$ computational model of facula production and destruction indicates that the observed number of craters with rim/wall faculae is consistent with the formation of floor faculae in large craters, dissemination by impact to form rim/wall faculae, and removal from the surface over $<1 \mathrm{Ga}$ timescales.

Data collectively point to a relatively modern formation or exposure of faculae, indicating that Ceres' surface remains active and the near surface may support brines in the present day. Faculae currently exposed on the surface continue to evolve due to the combined effects of space weathering and lateral mixing. Faculae were likely forming at even greater rates in the geologic past, and warmer subsurface temperatures and higher impact rates would have promoted near-surface brines with greater frequency than today. Rim/wall faculae expose these relicts of alteration as a window into Ceres' past aqueous alteration. Although the original formation of faculae is clearly associated with impact processes, it remains unclear whether portions of the subsurface are warm enough to exceed the eutectic temperatures of the progenitor species of faculae or if heating is instead provided directly by impacts. Future analysis of the floor faculae, and particularly observations of whether some or all floor faculae remain active in present day, may allow this distinction to be made.

\section{Acknowledgments}

We are indebted to the Dawn mission team for their efforts in collecting the data used in this paper, as well as to members of the FC and VIR teams for their helpful discussions. Thanks to M. Landis and V. Reddy for reviews that improved this manuscript. This material is based upon work supported by the National Science Foundation Graduate Research Fellowship Grant no. DGE-1144469. VIR is funded by the Italian Space Agency and was developed under the leadership of INAF-Istituto di Astrofisica e Planetologia Spaziali, Rome, Italy. The instrument was built by Selex-Galileo, Florence, Italy. This work was supported by the Italian Space Agency grant. Data used are available in the supplementary material or archived in the Planetary Data System (pds.nasa.gov).

\section{Supplementary materials}

Supplementary material associated with this article can be found, in the online version, at doi:10.1016/j.icarus.2017.10.014.

\section{References}

Ammannito, E., DeSanctis, M.C., Ciarniello, M., Frigeri, A., Carrozzo, F.G., Combe, J.-P., Ehlmann, B.L., Marchi, S., McSween, H.Y., Raponi, A., Toplis, M.J., Tosi, F., CastilloRogez, J.C., Capaccioni, F., Capria, M.T., Fonte, S., Giardino, M., Jaumann, R., Longobardo, A., Joy, S.P., Magni, G., McCord, T.B., McFadden, L.A., Palomba, E., Pieters, C.M., Polanskey, C.A., Rayman, M.D., Raymond, C.A., Schenk, P.M., Zambon, F., Russell, C.T., 2016. Distribution of phyllosilicates on the surface of Ceres. Science 353, 1006. doi:10.1126/science.aaf4279.

Bland, M.T., Raymond, C.A., Schenk, P.M., Fu, R.R., Kneissl, T., Hendrik, J., Pasckert, J.H., Hiesinger, H., Preusker, F., Park, R.S., Marchi, S., King, S.D., CastilloRogez, J.C., Russell, C.T., 2016. Composition and structure of the shallow subsurface of Ceres revealed by crater morphology. Nature Geoscience 9, 538-542. doi:10.1038/nature18290.

Bowling T.J., Ciesla F.J., Marchi S., Johnson B.C., Davison M., Castillo-Rogez J.C., De Sanctis M.C., Raymond C.A., Russell C.T., Impact induced heating of Occator Crater on asteroid 1 Ceres. In: Proceedings of the 47th Lunar and Planetary Science Conference, LPSC, 2016, Abstract 2268.

Buczkowski, D.L., Schmidt, B.E., Williams, D.A., Mest, S.C., Scully, J.E.C., Ermakov, A.I., Preusker, F., Schenk, P., Otto, K.A., Hiesinger, H., OBrien, D., Marchi, S., Sizemore, H., Hughson, K., Chilton, H., Bland, M., Byrne, S., Schorghofer, N., Platz, T., Jaumann, R., Roatsch, T., Sykes, M.V., Nathues, A., De Sanctis, M.C., Raymond, C.A., Russell, C.T., 2016. The geomorphology of Ceres. Science 353 aaf4332. doi:10.1126/science.aaf4332.

Carrozzo, F.G., De Sanctis, M.C., Ammannito, E., Castillo-Rogez, J., Ehlmann, B.L., Ciarniello, M., Marchi, S., Raponi, A., Stein, N., Tosi, F., Capaccioni, F., Capria, M.T. Fonte, S., Formisano, M., Frigeri, A., Giardino, M., Longobardo, A., Magni, G. Palomba, E., Zambon, F., Raymond, C.A., Russell, C.T., 2017. Distribution of carbonates on Ceres. Sci. Adv., in review.

Castillo-Rogez, J.C., McCord, T.B., 2010. Ceres' evolution and present state constrained by shape data. Icarus 205, 443-459. doi:10.1016/j.icarus.2009.04.008.

Ciarniello, M., De Sanctis, M.C., Ammannito, E., Raponi, A., Longobardo, A., Palomba, E., Carrozzo, F.G., Tosi, F., Li, J.-Y., Schröder, S.E., Zambon, F., Frigeri, A Fonte, S., Giardino, M., Pieters, C.M., Raymond, C.A., Russell, T., 2017. Spectrophotometric properties of dwarf planet Ceres from the VIR spectrometer on board the Dawn mission. Astron. Astrophys. 598, A130. doi:10.1051/0004-6361/ 201629490.

Combe, J.-P., McCord, T.B., Tosi, F., Ammannito, E., Carrozzo, F.G., De Sanctis, M.C., Raponi, A., Byrne, S., Landis, M.E., Hughson, K.H.G., Raymond, C.A., Russell, C.T., 2016. Detection of local $\mathrm{H}_{2} \mathrm{O}$ exposed at the surface of Ceres. Science 353, aaf3010. doi:10.1126/science.aaf3010.

Combe, J.P., Raponi, A., Tosi, F., De Sanctis, M.C., Ammannito, E., Carrozzo, F.G., Landis, M.E., Byrne, S., Carsety, U., Schröder, S., Platz, T., Ruesch, O., Hughson, K., McCord, T.B., Singh, S., Johnson, K., Zambon, F., Pieters, C.M., Raymond, C.A., Russell, C.T., 2017. Exposed $\mathrm{H}_{2} \mathrm{O}$-rich areas on Ceres detected by Dawn. In: Proceedings of 48th Lunar and Planetary Science Conference, LPSC Abstract 2568.

Denevi, B.W., Blewett, D.T., Buczkowski, D.L., Capaccioni, F., Capria, M.T., De Sanctis, M.C., Garry, W.B., Gaskell, R.W., Le Corre, L., Li, J.Y., Marchi, S., 2012. Pitted terrain on Vesta and implications for the presence of volatiles. Science 338 (6104), 246-249. doi:10.1126/science.1225374.

De Sanctis, M.C., Ammannito, E., Raponi, A., Marchi, S., McCord, T.B., McSween, H.Y Capaccioni, F., Capria, M.T., Carrozzo, F.G., Ciarniello, M., Longobardo, A., Tosi, F., Fonte, S., Formisano, M., Frigeri, A., Giardino, M., Magni, G., Palomba, E., Turrini, D., Zambon, F., Combe, J.-P., Feldman, W., Jaumann, R., McFadden, L.A., Pieters, C.M., Prettyman, T., Toplis, M., Raymond, C.A., Russell, C.T., 2015. Ammoniated phyllosilicates with a likely outer Solar System origin on (1) Ceres Nature 528, 241-244. doi:10.1038/nature16172.

De Sanctis, M.C., Raponi, A., Ammannito, E., Ciarniello, M., Toplis, M.J., McSween, H.Y., Castillo-Rogez, J.C., Ehlmann, B.L., Carrozzo, F.G., Marchi, S., Tosi, F., Zambon, F., Capaccioni, F., Capria, M.T., Fonte, S., Formisano, M., Frigeri, A., Giardino, M., Longobardo, A., Magni, G., Palomba, E., McFadden, L.A., Pieters, C.M., Jaumann, R., Schenk, P., Mugnuolo, R., Raymond, C.A., Russell, C.T., 2016. Bright carbonate deposits as evidence of aqueous alteration on (1) Ceres. Nature 536, 1-4. doi:10.1038/nature18290.

Ermakov, A.I., Fu, R.R., Castillo-Rogez, J.C., Raymond, C.A., Park, R.S., Preusker, F., Russell, C.T. Smith, D.E., Zuber, M.T. 2017. Constraints on Ceres' internal structure and evolution from its shape and gravity measured by the Dawn spacecraft, J. Geophys. Res., https://doi.org/10.1002/2017je005302.

Hayne, P.O., Aharonson, O., 2015. Thermal stability of ice on Ceres with rough topography. J. Geophys. Res. Planets. 120, 1567-1584. doi:10.1002/2015JE004887.

Hiesinger, H., Marchi, S., Schmedemann, N., Schenk, P., Pasckert, J.H., Neesemann, A., OBrien, D.P., Kneissl, T., Ermakov, A.I., Fu, R.R., Bland, M.T., Nathues, A Platz, T., Williams, D.A., Jaumann, R., Castillo-Rogez, J.C., Ruesch, O., Schmidt, B., Park, R.S., Preusker, F., Buczkowski, D.L., Russell, C.T., Raymond, C.A., 2016. Cratering on Ceres: implications for its crust and evolution. Science 353. doi:10. 1126/science.aaf4759, aaf4759-aaf4759.

Jaumann, R., Stephan, K., Krohn, K., Matz, K.D., Otto, K., Neumann, W., Kneissl, T. Schmedemann, N., Schröder, S., Tosi, F., De Sanctis, M.C., Preusker, F. Buczkowski, D., Capacioni, F., Carsenty, U., Elgner, S., von der Gathen, I., Giebner, T., Hiesinger, H., Hoffmann, M., Kersten, E., Li, J.Y., McCord, T.B., McFadden, L., Mottola, S., Nathues, A., Neesemann, A., Raymond, C., Roatsch, T., Russell, C.T., Schmidt, B., Schulzeck, F., Wagner, R., Williams, D.A., 2016. Age-dependent morphological and compositional variations on Ceres. In: Proceedings of the 47th Lunar and Planetary Science Conference, LPSC Abstract 1455.

Jaumann, R., Preusker, F., Krohn, K., von der Gathen, I., Stephan, K., Matz, K.D. Elgner, S., Otto, K., Schmedemann, N., Nessemann, A., Roatsch, T., Kersten, E., Schröder, S., Schulzneck, F., Tosi, F., De Sanctis, M.C., Buczkowski, D., Scully, J.E.C. Hiesinger, H., Raymond, C., Russell, C.T., Stein, N.T., Williams, D.A., Ruesch, O. Schenk, P., 2017. Topography and geomorphology of the interior of Occator Crater on Ceres. In: Proceedings of the 48th Lunar and Planetary Science Conference, LPSC Abstract 1440.

Jozwiak, L.M., Head, J.W., Zuber, M.T., Smith, D.E., Neumann, G.A., 2012. Lunar floorfractured craters: classification, distribution, origin and implications for magmatism and shallow crustal structure. J. Geophys. Res. E: Planets 117, 1-23. doi:10.1029/2012JE004134.

King, T.V., Clark, R.N., Calvin, W.M., Sherman, D.M., Brown, R.H. 1992. Evidence for ammonium-bearing minerals on Ceres. Science 255, 1551-1553. doi:10.1126 science.255.5051.1551

Li, J.Y., Reddy, V., Nathues, A., Corre, L.Le, Izawa, M.R.M., Cloutis, E.A., Sykes, M.V., Carsenty, U., Castillo-Rogez, J.C., Hoffmann, M., Jaumann, R., Krohn, K., Mottola, S., Prettyman, T.H., Schaefer, M., Schenk, P.M., Schröder, S.E., Williams, D.A., Smith, D.E., Zuber, M.T., Konopliv, A.S., Park, R.S., Raymond, C.A., Russell, C.T. 
2016. Surface Albedo and Spectral Variability of Ceres. Astrophys. J. 817, L22. doi:10.3847/2041-8205/817/2/L22.

McCord, T.B., Combe, J.-Ph., McSween, H., Jaumann, R., Reddy, V., Tosi, F., Williams, D., Blewett, D.T., Turrini, N.D., Palomba, E., Raymond, C.A., Russell, C.T., Li, J-Y., Pieters, C.M., De Sanctis, M.C., Ammannito, E., Capria, M.T., Le Corre, L., Longobardo, A., Nathues, A., the Dawn Team, 2012. Dark material on Vesta: adding carbonaceous volatile-rich materials to planetary surfaces. Nature 491, 83-86. doi:10.1038/nature11561.

Nathues, A., Hoffmann, M., Schaefer, M., Le Corre, L., Reddy, V., Platz, T., Cloutis, E.A., Christensen, U., Kneissl, T., Li, J.-Y., Mengel, K., Schmedemann, N., Schaefer, T., Russell, C.T., Applin, D.M., Buczkowski, D.L., Izawa, M.R.M., Keller, H.U., O'Brien, D.P., Pieters, C.M., Raymond, C.A., Ripken, J., Schenk, P.M., Schmidt, B.E., Sierks, H., Sykes, M.V., Thangjam, G.S., Vincent, J.-B., 2015. Sublimation in bright spots on (1) Ceres. Nature 528, 237-240. doi:10.1038/nature15754.

Nathues, A., Hoffman, M., Platz, T., Thangjam, G.S., Cloutis, E.A., Reddy, V., Le Corre, L., Li, J.-Y., Mengel, K., Rivkin, A., Applin, D.M., Schaefer, M., Christensen, U., Sierks, H., Ripken, J., Schmidt, B.E., Hiesinger, H., Sykes, M.V., Sizemore, H.G., Preusker, F., Russell, C.T., 2016. FC colour images of dwarf planet Ceres reveal a complicated geological history. Planet. Space Sci. 134, 122-127. doi:10.1016/j.pss.2016.10.017.

Nathues, A., Platz, T., Thangjam, G., Hoffmann, M., Mengel, K., Cloutis, E.A., Le Corre, L., Reddy, V., Kallisch, J., Crown, D.A., 2017a. Evolution of Occator Crater on (1) Ceres. Astron. J. 153, 112. doi:10.3847/1538-3881/153/3/112.

Nathues, A., Platz, T. Hoffmann, M., Thangjam, G., Cloutis, E.A., Applin, D.M., Le Corre, L., Reddy, V., Mengel, K., Protopapa, S., 2017b. Oxo Crater on (1) Ceres - geologic history and the role of water ice. Astrophys. J. 154, 84. doi:10.3847/ 1538-3881/aa7a04

Neesemann, A., van Gasselt, S., Schmedemann, N., Marchi, S., Walter, S.H.G., Preusker, F., Michael, G.G., Kneissl, T., Hiesinger, H., Jaumann, R., Roatsch, T., Raymond, C.A., Russell, C.T., The various ages of Occator crater, Ceres: Results of a comprehensive synthesis approach. Submitted to Icarus. This special issue.

Palomba, E., Longobardo, A., De Sanctis, M.C., Stein, N.T., Ehlmann, B., Galiano, A. Raponi, A., Ciarniello, M., Ammannito, E., Cloutis, E., Carrozzo, F.G., Capria, M.T., Stephan, K., Zambon, F., Tosi, F., Raymond, C.A., Russell, C.T., 2017. Compositional differences among Bright Spots on the Ceres surface. Icarus doi:10.1016/j.icarus. 2017.09.020.

Pasckert, J.H., Hiesinger, H., Williams, D.A., Crown, D.A., Mest, S.C., Buczkowski, D.L., Scully, J.E.C., Schmedemann, N., Jaumann, R., Roatsch, T., Preusker, F., Naß, A., Nathues, A., Hoffmann, M., Schäfer, M., De Sanctis, M.C., Raymond, C.A., Russell, C.T., 2016. Geologic mapping of the Ac-H-2 Coniraya quadrangle of Ceres from NASA's Dawn mission. In: Proceedings of 47th Lunar and Planetary Science Conference, LPSC Abstract 1450.

Pasckert, J.H., Hiesinger, H., Ruesch, O., Williams, D.A., Naß, A., Kneissl, T., Mest, S.C., Buczkowski, D.L., Scully, J.E.C., Schmedemann, N., Jaumann, R., Roatsch, T., Preusker, F., Nathues, A., Hoffmann, M., Schäfer, M., De Sanctis, M.C., Raymond, C.A., Russell, C.T., 2017. Geologic mapping of the Ac-2 Coniraya quadrangle of Ceres from NASA's Dawn mission: Implications for a heterogeneously composed crust. Icarus doi:10.1016/j.icarus.2017.06.015.

Pieters, C.M., Ammannito, E., Ciarniello, M., De Sanctis, M.C., Hoffmann, M., Jaumann, R., McCord, T.B., McFadden, L.A., Mest, S., Nathues, A., Raponi, A., 2016. Surface processes and space weathering on Ceres. In: Proceedings of 47th Lunar and Planetary Science Conference, LPSC Abstract 1383.

Pieters, C.M., Noble, S.K., 2016. Space weathering on airless bodies. J. Geophys. Res.: Planets 121, 1865-1884. doi:10.1002/2016JE005128.

Prettyman, T.H., Yamashita, N., Toplis, M.J., McSween, H.Y., Schorghofer, N., Marchi, S., Feldman, W.C., Castillo-Rogez, J., Forni, O., Lawrence, D.J., Ammannito, E., Ehlmann, B.L., Sizemore, H.G., Joy, S.P., Polanskey, C.A., Rayman, M.D., Raymond, C.A., Russell, C.T., 2016. Extensive water ice within Ceres aqueously altered regolith: Evidence from nuclear spectroscopy. Science 6765, 1-11. doi:10. 1126/science.aah6765.
Reddy, V., Le Corre, L., O’Brien, D.P., Nathues, A., Cloutis, E.A., Durda, D.D., Bottke, W.F., Bhatt, M.U., Nesvorny, D., Buczkowski, D., Scully, J.E.C., Palmer, E.M., Sierks, H., Mann, P.J., Becker, K.J., Beck, A.W., Mittlefehldt, D., Li, J-Y., Gaskell, R. Russell, C.T., Gaffey, M.J., McSween, H.Y., McCord, T.B., Combe, J-P., Blewett, D., 2012. Delivery of dark material to Vesta via carbonaceous chondritic impacts. Icarus 221 (2), 544-559. doi:10.1016/j.icarus.2012.08.011.

Ruesch, O., Platz, T., Schenk, P., McFadden, L.A., Castillo-Rogez, J.C., Quick, L.C., Byrne, S., Preusker, F., OBrien, D.P., Schmedemann, N., Williams, D.A., Li, J.Y., Bland, M.T., Hiesinger, H., Kneissl, T., Neesemann, A., Schaefer, M., Pasckert, J.H., Schmidt, B.E., Buczkowski, D.L., Sykes, M.V, Nathues, A., Roatsch, T., Hoffmann, M., Raymond, C.A., Russell, C.T., 2016. Cryovolcanism on Ceres. Science 353, aaf4286. doi:10.1126/science.aaf4286.

Schmedemann, N., Kneissl, T., Neesemann, A., Stephan, K., Jaumann, R., Krohn, K. Michael, G.G., Matz, K.D., Otto, K.A., Raymond, C.A., Russell, C.T., 2016. Timing of optical maturation of recently exposed material on Ceres. Geophys. Res. Lett. 43, 11,987-11,993. doi:10.1002/2016GL071143.

Schröder, S.E., Carsenty, U., Ciarniello, M. Jaumann, R., Li, J.Y, Longobardo, A Palmer, E., Pieters, C., Preusker, F., Raymond, C.A., Russell, C.T., 2017. Resolved spectrophotometric properties of the Ceres surface from Dawn Framing Camera images. Icarus 228, 201-225. doi:10.1016/j.icarus.2017.01.026.

Schultz, P.H., 1976. Floor-fractured lunar craters. Moon 15, 241-273. doi:10.1007| BF00562240.

Sierks, H., Keller, H.U., Jaumann, R., Michalik, H., Behnke, T., Bubenhagen, F., Büttner, I., Carsenty, U., Christensen, U., Enge, R., Fiethe, B., Gutiérrez Marqués, P., Hartwig, H., Krüger, H., Kühne, W., Maue, T., Mottola, S., Nathues, A., Reiche, K.U., Richards, M.L., Roatsch, T., Schröder, S.E., Szemerey, I., Tschentscher, M., 2011. The Dawn framing camera. Space Sci. Rev. 163, 263-327. doi:10.1007/ s11214-011-9745-4.

Sizemore, H.G., Platz, T., Schorghofer, N., Prettyman, T.H., De Sanctis, M.C., Crown, D.A., Schmedemann, N., Neesemann, A., Kneissl, T., Marchi, S., Schenk, P.M., Bland, M.T., Schmidt, B.E., Hughson, K.H.G., Tosi, F., Zambon, F., Mest, S.C., Yingst, R.A., Williams, D.A., Russell, C.T., Raymond, C.A., 2017. Pitted terrains on (1) Ceres and implications for shallow subsurface volatile distribution. Geophys. Res. Lett. 44, 6570-6578. doi:10.1002/2017GL073970.

Stephan, K., Jaumann, R., Krohn, K., Schmedemann, N., Zambon, F., Tosi, F., Carrozzo, F.G., McFadden, L.A., Otto, K., De Sanctis, M.C., Ammannito, E., Matz, K.D., Roatsch, T., Preusker, F., Raymond, C.A., Russell, C.T., 2017. An investigation of the bluish material on Ceres. Geophys. Res. Lett. 44, 1660-1668. doi:10.1002 2016 GL071652.

Thangjam, G., Hoffmann, M., Nathues, A., Li, J.Y., Platz, T., 2016. Haze at occator crater on dwarf planet ceres. Astrophys. J. Lett. 833, L25. doi:10.3847/ 2041-8213/833/2/L25.

Zambon, F., Raponi, A., Tosi, F., De Sanctis, M.C., McFadden, L.A., Carrozzo, F.G., Longobardo, A., Ciarniello, M., Krohn, K., Stephan, K., Palomba, E., Pieters, C.M., Ammannito, E., Russell, C.T., Raymond, C.A., 2017. Spectral analysis of Ahuna Mons from Dawn mission's visible-infrared spectrometer. Geophys. Res. Lett. 44, 97104. doi:10.1002/2016GL071303. 